\title{
MODULAR SYMBOLS AND THE TOPOLOGICAL NONRIGIDITY OF ARITHMETIC MANIFOLDS
}

\author{
STANLEY CHANG, SHMUEL WEINBERGER
}

\begin{abstract}
In this paper we address the existence of smooth manifolds proper homotopy equivalent to nonuniform arithmetic manifolds $M=\Gamma \backslash G / K$ that are not homeomorphic to it. While the manifolds $M$ are properly rigid if $\operatorname{rank}_{\mathbb{Q}}(\Gamma) \leq 2$, we show that the so-called "virtual structure group" has infinite rank as a $\mathbb{Q}$-vector space if $\operatorname{rank}_{\mathbb{Q}}(\Gamma) \geq 4$.
\end{abstract}

\section{Contents}

1. The surgery set-up 5

2. Lattices commensurable with $\mathrm{SL}_{n}(\mathbb{Z})$ for $n \geq 5 \quad 6$

3. Proof for large $\mathbb{Q}$-rank $\quad 9$

4. The Hermitian symmetric case 11

5. The virtual structure set $\quad 13$

6. Computations 16

$\begin{array}{ll}\text { References } & 26\end{array}$

The Borel conjecture asserts that closed aspherical manifolds are topologically rigid; i.e. if $M$ is closed and aspherical and $f: M^{\prime} \rightarrow M$ is a homotopy equivalence, then $f$ is homotopic to a homeomorphism. This statement is a topological analogue of Mostow rigidity, which in its simplest version asserts that, in the situation where both manifolds are hyperbolic of dimension at least 3 , such a map $f$ is in fact homotopic to an isometry. The Borel conjecture has been verified in locally symmetric situations by Farrell-Jones [24] (aside from dimension four cases) and for many non-classical aspherical manifolds by Bartels-Lück [6].

In light of Prasad's extension of Mostow rigidity from uniform to non-uniform lattices [42], it becomes natural to inquire whether there is a proper analogue to the Borel conjecture. Here we define a noncompact manifold $M$ to be properly rigid if every proper homotopy equivalence $f: M^{\prime} \rightarrow M$ is properly homotopic to a homeomorphism. Already in 1982, Farrell and Hsiang [23] showed that, for $n>176$,

Date: July 4, 2013.

Key words and phrases. arithmetic manifolds, virtual structure set, modular symbols.

Research partially supported by NSF Grant DMS-9971657 and a grant from the Wellesley Brachman-Hoffman Fellowship. 
if $\Gamma \subseteq \mathrm{SL}_{n}(\mathbb{Z})$ is torsion-free, then $\Gamma \backslash \mathrm{SL}_{n}(\mathbb{R}) / \mathrm{SO}_{n}(\mathbb{R})$ is not properly rigid. In our earlier paper [18] we proved the following.

Theorem 0.1. Let $M$ be a locally symmetric manifold $\Gamma \backslash G / K$, where $G$ is a semisimple Lie group, $K$ is its maximal compact subgroup and $\Gamma$ is an irreducible lattice.

(1) If $\Gamma$ is not arithmetic then it is properly rigid.

(2) If $\Gamma$ is arithmetic and its $\mathbb{Q}$-rank at most 2, then the twisted Borel conjecture for $\Gamma$ implies its proper rigidity.

(3) If $\Gamma$ has $\mathbb{Q}$-rank at least 3 then $M$ has a finite-sheeted cover $M^{\prime}=\Gamma^{\prime} \backslash G / K$ for which proper rigidity fails.

The proofs of these statements use a combination of methods from surgery, superrigidity, and strong approximation for linear groups. The recent paper of BartelsLück-Reich-Rüping [7] proves the relevant twisted Borel conjecture that establishes (2) unconditionally. Notice that (3) shows that the Borel conjecture fails systematically in the noncompact proper setting.

There are a number of issues that the above theorem and its proof do not resolve. We will introduce some terminology to address some new ideas.

Definition 0.2. Let $M$ be a noncompact manifold. We say that $M$ is (properly topologically) strongly nonrigid if there is a element $f: N \rightarrow M$ in $S^{T o p}(M)$ such that $N$ is not homeomorphic to $M$. In this case, we say that $N$ is an exotic version of $M$. If $f: N \rightarrow M$ is a nontrivial element in $S^{T o p}(M)$ with $N$ homeomorphic to $M$, we say that $f$ is exotic; i.e. $f$ is not properly homotopic to a homeomorphism. The trivial element of $S^{T o p}(M)$ is merely the class containing the identity map $i: M \rightarrow M$.

Let $M=\Gamma \backslash G / K$ be a locally symmetric manifold with $\Gamma$ irreducible and let $M^{\prime}=$ $\Gamma^{\prime} \backslash G / K$ be a finite-sheeted cover which is properly nonrigid. We can ask the following questions.

(a) Are there any exotic versions of $M^{\prime}$ in $S^{T o p}\left(M^{\prime}\right)$, or is it the case that every $f: N \rightarrow M^{\prime}$ in $S^{T o p}\left(M^{\prime}\right)$ is merely exotic?

(b) If exotic versions of $M^{\prime}$ exist, do they have smooth structures?

(c) Suppose that $f: N \rightarrow M^{\prime}$ is a nontrivial element of $S^{T o p}\left(M^{\prime}\right)$. Is there finite-sheeted lift $\widetilde{M^{\prime}}$ of $M^{\prime}$ such that the induced lift $\widetilde{f}: \widetilde{N} \rightarrow \widetilde{M^{\prime}}$ is trivial $S^{T o p}\left(\widetilde{M^{\prime}}\right)$ ?

We can produce a reducible example related to question (c). Let $\Pi$ be a product of three noncompact surfaces. Then for any cover $\Pi^{\prime}$, the set of manifolds proper homotopy equivalent to $\Pi^{\prime}$ is finite, but all of these manifolds become homeomorphic upon passing to an appropriate further finite cover. Although the examples here are all smoothable, the method of the authors' previous work [18], which makes use of topological characteristic classes, is definitely topological in nature. Moreover, 
all of the examples in [18] actually do become trivial on passing to a further finite cover. Amusingly, they are all nontrivial upon passing to odd-fold covers.

The following theorem addresses questions (a) and (b) in the irreducible case.

Theorem 0.3. Let $M=\Gamma \backslash G / K$ be an arithmetic manifold with $\Gamma$ irreducible and $\mathbb{Q}$-rank at least 6. Then for all integers $A$, there is a finite-sheeted cover $N_{A}$ of $M$ such that the number of smooth exotic versions of $N_{A}$ is at least $A$. As a consequence, the proper topological structure set $S^{T o p}\left(N_{A}\right)$ can be made arbitrarily large. In addition, these exotic versions can be distinguished from each other by their first Pontrjagin class.

The proof of the last statement above implicitly makes use of a refinement of Novikov's theorem on the topological invariance of rational Pontrjagin classes $p_{i}$. Because of the rational equivalence of the map $F / O \rightarrow F / T o p$, in each dimension $d$, there is a positive integer $C_{d}$ so that $C_{d} p_{d}$ is a topological invariant. Therefore, we can make use of torsion elements in $H^{4}(N ; \mathbb{Z})$ to distinguish topological manifolds.

The goal of this paper is to strengthen the nonrigidity of Theorem 0.1(3) by addressing the questions above. By using higher Pontrjagin classes and modular symbols, we obtain the following substantial improvement, showing that strong nonrigidity holds when the $\mathbb{Q}$-rank is at least 4 .

Theorem 0.4. Let $\Gamma$ be a lattice in a semisimple algebraic group $G$ over $\mathbb{Q}$ with $\mathbb{Q}$-rank at least 4. Let $K$ be a maximal compact subgroup of $G$. Then there is $\Gamma^{\prime} \leq \Gamma$ such that $\Gamma^{\prime} \backslash G / K$ is strongly nonrigid.

The proof of Theorem 0.3 is a case-by-case argument while that of Theorem 0.4 is a general argument.

Among the examples presented in this paper is the special case of Hermitian locally symmetric spaces. Such a space $M$ is a Riemannian symmetric space with a parallel complex structure with respect to which the Riemannian metric is Hermitian. Here we exploit the projectivity of the Baily-Borel compactification and the observation by Jost-Yau [32] that the codimension of the singular set is usually reasonably large. With these notions, we can prove that, when the complex dimension is at least 3, the space $M$ is properly nonrigid. The converse is also true.

Theorem 0.5. If $M=\Gamma \backslash G / K$ is a Hermitian locally symmetric manifold of real dimension at least 5, then the following are equivalent:

(1) The $\mathbb{Q}$-rank of $\Gamma$ is at least 3.

(2) The space $M$ is properly topologically nonrigid.

If these conditions are satisfied, then infinitely many elements in $S^{T o p}(M)$ are in fact detected by a rational topological version of the first Pontrjagin class in $H^{4}(M ; \mathbb{Q})$. Also in this case, the space $M$ is properly nonrigid among smooth manifolds (where the conclusion is still homeomorphism). 
In other words, for the case of Hermitian symmetric manifolds, Theorem 0.5 strengthens Theorem 0.1 in all of the ways discussed, without requiring that any covers be taken.

Having established the nonvanishing of the proper structure sets, we are interested to know whether they grow, in any sense, when the symmetric space becomes larger. This notion suggests that we can define a set $S^{\text {virt }}(M)$, a virtual structure group of a manifold $M$. Neither the methods used for Hermitian symmetric spaces nor the argument of Farrell-Hsiang can be used to bound this object, since these techniques are based on cohomology classes arising from the compact dual, and in this case there are only finitely many. However, using modular symbols we can prove that $S^{\text {virt }}(\Gamma \backslash G / K)$ is not only nontrivial, but has infinite rational rank as a $\mathbb{Q}$-vector space. We remark additionally that $S^{T o p}(\Gamma \backslash G / K)$ has a natural abelian group structure. Note that in this theorem we do not assume that $\Gamma \backslash G / K$ is irreducible.

Theorem 0.6. Suppose that $\Gamma \backslash G / K$ is an arithmetic manifold with $\mathbb{Q}$-rank at least 4. Then $S^{\text {virt }}(\Gamma \backslash G / K) \otimes \mathbb{Q}$ is an infinite-dimensional $\mathbb{Q}$-vector space.

The size of the group $S^{T o p}(\Gamma \backslash G / K) \otimes \mathbb{Q}$ usually grows sublinearly in the covolume of $\Gamma$. The methods of $L^{2}$-cohomology imply that linear growth occurs in a tower precisely when $\operatorname{dim}(G / K)$ is a multiple of 8 and $\operatorname{rank}_{\mathbb{C}} G=\operatorname{rank}_{\mathbb{C}} K$. The characterization should either be interpreted as the condition for the existence of discrete series representations, or equivalently for the compact dual of $G / K$ to have nonzero Euler characteristic (which is then the ratio of the order of the Weyl groups). However, even when the growth is sublinear, when one passes up the congruence tower, the ranks of these abelian groups do grow like a positive power of the volume.

Our methods give some information even if the $\mathbb{Q}$-rank is 3. For example, if $\mathcal{O}_{\mathbb{F}}$ is a ring of algebraic integers, then the arithmetic manifold associated to $\mathrm{SL}_{n}\left(\mathcal{O}_{\mathbb{F}}\right)$ is nonrigid, with infinitely generated virtual structure set. However, there are different phenomena that arise in general. For example, the virtual structure set vanishes for product lattices in $\mathrm{SL}_{2}(\mathbb{R}) \times \mathrm{SL}_{2}(\mathbb{R}) \times \mathrm{SL}_{2}(\mathbb{R})$, as mentioned previously. It would be interesting to clarify the $\mathbb{Q}$-rank 3 situation fully.

The paper is organized as follows. The first section gives some basic remarks about the surgery theory required in this examination. Section 2 discusses $\mathrm{SL}_{n}(\mathbb{Z})$ from several points of view and gives the proof of Theorem 0.3 in this case. (Note that, when $\mathcal{O}$ is a number ring, we obtain the optimal results of nonrigidity for $\operatorname{SL}_{n}(\mathcal{O})$ when $n \geq 4$.) The modifications for the more general case are given in section 3 . The key idea is to identify deep lattices of a simple algebraic group $G$ over $\mathbb{Q}$ that represents nonzero cycles in appropriate homology groups. Ideas of this sort appear in papers of Lee-Szczarba [34], Charney [19] and Church-Farb [21]. We also quickly show, using a Bockstein argument, that the elements constructed in [18] by strong approximation represents the zero element in its virtual structure set. Section 4 discusses the Hermitian locally symmetric case, while section 5 defines the virtual 
structure set of an arithmetic manifold and proves its infinite size when the $\mathbb{Q}$-rank is at least 4 . The last section gives a list of the numerical calculations required in section 5 .

Most of this paper was done while the authors visited MSRI in Fall 2012 for the special semester on Quantitative Topology. We thank MSRI for its hospitality. We also thank Tom Church and Benson Farb for useful conversations. We give special acknowledgment to David Witte Morris for helping us the calculations involved in Section 6 of the paper.

\section{THE SURGERY SET-UP}

As given in Wall [48] the surgery exact sequence for a given compact manifold ${ }^{1} M$ of dimension $n$ is given by

$$
\cdots \rightarrow L_{n-1}\left(\mathbb{Z} \pi_{1}(M)\right) \rightarrow S^{T o p}(M) \rightarrow[M: F / T o p] \rightarrow L_{n}\left(\mathbb{Z} \pi_{1}(M)\right),
$$

where the $L_{*}\left(\mathbb{Z} \pi_{1}(M)\right)$ denote the Wall $L$-groups and $S^{T o p}(M)$ is the topological structure set, i.e the collection of pairs $(N, f)$, where $N$ is an $n$-manifold and $f: N \rightarrow M$ is a homotopy equivalence, modulo $h$-cobordism. ${ }^{2}$

Let $N=\Gamma \backslash G / K$ be a locally symmetric space formed as the double quotient of an irreducible Lie group $G$ with its maximal compact subgroup $K$ and an arithmetic lattice $\Gamma$. As observed by Block and Weinberger [9], this $N$ can be compactified (via the Borel-Serre compactification) to a $\pi-\pi$ manifold $\bar{N}$ with boundary when the $\mathbb{Q}$-rank is at least 3. By a $\pi-\pi$ manifold we mean a manifold whose fundamental group coincides with the fundamental group of its boundary. This observation of Block-Weinberger follows from the identification of the homotopy type of the $\Gamma$-cover of the boundary with a wedge of $(q-1)$-spheres using the Solomon-Tits theorem [10]. According to Siebenmann's thesis [46], any manifold that is properly homotopy equivalent to $M$ will have the same property. By way of the $h$-cobordism theorem, one can show that any such manifold has a unique compactification so that the extension of the proper homotopy equivalence to the compactification is a simple homotopy equivalence. We can then identify the structure set $S^{T o p}(\bar{N})$ with the proper structure set $S^{T o p}(N)$. (Here we suppress the $p$ for proper in the notation when it is clear that the space is noncompact.)

Thus by Wall's $\pi-\pi$ theorem [48], the structure set $S^{T o p}(\bar{N})$ of $\bar{N}$ is isomorphic to $[\bar{N}, F / T o p]$. Since $F /$ Top $_{(2)} \cong \prod_{n=1}^{\infty} K\left(\mathbb{Z}_{2}, 4 n-2\right) \times \prod_{m=1}^{\infty} K(\mathbb{Z}, 4 m)$ and $F / T o p[1 / 2] \cong B O[1 / 2]$ (see Madsen-Milgram [36]), we then have

$$
\begin{aligned}
S^{T o p}(N)_{(2)} & =S(\bar{N})_{(2)}=[\bar{N}, F / \text { Top }]_{(2)}=[N, F / \text { Top }]_{(2)} \\
& =\prod_{n=1}^{\infty} H^{4 n-2}\left(N ; \mathbb{Z}_{2}\right) \times \prod_{m=1}^{\infty} H^{4 m}\left(N ; \mathbb{Z}_{(2)}\right)
\end{aligned}
$$

\footnotetext{
${ }^{1}$ We ignore issues of orientation.

${ }^{2}$ In our situation, the difference between $h$-cobordism and homeomorphism can be safely ignored.
} 
and $S^{T o p}(M)[1 / 2] \cong K O^{0}(X)[1 / 2]$. To prove that the proper structure set is nontrivial, it suffices to show that some cohomology group $H^{4 n-2}\left(N ; \mathbb{Z}_{2}\right)$ or $H^{4 m}\left(N ; \mathbb{Z}_{(2)}\right)$ is nontrivial.

In [18] we proved that any $M=\Gamma \backslash G / K$ with $\mathbb{Q}$-rank at least 3 has a lift $N$ for which $H^{2}\left(N ; \mathbb{Z}_{2}\right)$ is nontrivial, thereby showing that $S^{T o p}(N)$ is also nontrivial. The topological nonrigidity of $N$, however, does not rule out the case in which each nontrivial element $N^{\prime}$ of $S^{T o p}(N)$ is merely a homeomorphic copy of $N$ equipped with a proper homotopy equivalence $f: N^{\prime} \rightarrow N$ that is not proper homotopic to a homeomorphism. If there is $N^{\prime}$ homotopy equivalent to $N$ but not homeomorphic, we say that $N^{\prime}$ is an exotic version of $N$. One goal of this paper is to identify exotic versions of arithmetic manifolds $\Gamma \backslash G / K$.

To identify such exotic types, we shall give several different methods, all of which exploit the $H^{4 i}\left(-; \mathbb{Z}_{(2)}\right)$ summand. One method is associated with $i=1$ and uses a topologically invariant Pontrjagin class $p_{1}$ to obtain flexibility. In general these obstructions will be torsion elements and can vanish in the cohomology groups associated to higher covers. Using varying $i$ and various rational cohomology classes we will be able to identify infinitely many obstructions of a fixed level and elements that persist in the cohomology groups associated to deeper sublattices.

\section{Lattices COMmensurable With $\mathrm{SL}_{n}(\mathbb{Z})$ FOR $n \geq 5$}

In this section we will assume that $n \geq 5$. Our arguments can readily be modified to $\mathrm{SL}_{n}(\mathcal{O})$, where $\mathcal{O}$ is any ring of integers. If $R$ is a ring, denote by $M_{n}(R)$ the collection of $n \times n$ matrices with entries in $R$. Denote by $\mathbb{Z}_{r}$ the cyclic group of order $r$. If $A \in M_{n}(\mathbb{R})$ and $r \geq 2$ is a positive integer, we will denote by $[A]_{r}$ its projection in $M_{n}\left(\mathbb{Z}_{r}\right)$. Let $p$ be a prime and consider the (principal) congruence subgroup $\Gamma_{p}$ of $\mathrm{SL}_{n}(\mathbb{Z})$ given by

$$
\Gamma_{p} \rightarrow \mathrm{SL}_{n}(\mathbb{Z}) \rightarrow \mathrm{SL}_{n}\left(\mathbb{Z}_{p}\right)
$$

In other words we have $\Gamma_{p}=\left\{A \in \mathrm{SL}_{n}(\mathbb{Z}):[A]_{p}=I\right\}$. Since the $\mathbb{Q}$-rank of $\mathrm{SL}_{n}(\mathbb{Z})$ in $\mathrm{SL}_{n}(\mathbb{R})$ is $n-1$, it follows that the $\mathbb{Q}$-rank of $\Gamma_{p}$ in $\mathrm{SL}_{n}(\mathbb{R})$ is at least 3. Our previous theorem states that some lift of $M_{p}=\Gamma_{p} \backslash \mathrm{SL}_{n}(\mathbb{R}) / \mathrm{SO}_{n}(\mathbb{R})$ is properly nonrigid, but does not give any additional information about $M_{p}$ itself. Note that, at the moment, we are working with general $p$ but later we will choose $p$ more precisely.

We will restrict ourselves first to the discussion of $n=5$. The reader will see that the general case is treated in exactly the same manner. As a matter of notation, 
let the matrix

$$
\left(\begin{array}{lllll}
1 & a & b & c & d \\
0 & 1 & e & f & g \\
0 & 0 & 1 & 0 & 0 \\
0 & 0 & 0 & 1 & 0 \\
0 & 0 & 0 & 0 & 1
\end{array}\right)
$$

be denoted by $\left\|\begin{array}{llll}a & b & c & d \\ 1 & e & f & g\end{array}\right\|$. Note that all matrices in $\mathrm{SL}_{n}(\mathbb{Z})$ of the form $\left\|\begin{array}{cccc}a & b & 0 & 0 \\ 1 & e & 0 & 0\end{array}\right\|$ can be identified with the Heisenberg group and, for all primes $p$, those matrices of the form $\left\|\begin{array}{cccc}p a & p b & 0 & 0 \\ 1 & p e & 0 & 0\end{array}\right\|$ form the $p$-multiple Heisenberg group Heis $_{p}$. We distinguish two other subgroups of $\mathrm{SL}_{n}(\mathbb{Z})$. The subgroup of all elements of the form $\left\|\begin{array}{cccc}0 & p b & p c & p d \\ 1 & 0 & 0 & 0\end{array}\right\|$ will be denoted $A_{p}$, and the subgroup of all elements of the form $\left\|\begin{array}{cccc}p a & p b & p c & p d \\ 1 & p e & 0 & 0\end{array}\right\|$ will be denoted Heis ${ }_{p}^{+}$. Note that Heis ${ }_{p}^{+}$, $A_{p}$ and Heis $_{p}$ are all subgroups of $\Gamma_{p}$. In addition, denote by $\langle p b\rangle,\langle p c\rangle$ and $\langle p d\rangle$ the infinite cyclic subgroups generated by $\left\|\begin{array}{cccc}0 & p b & 0 & 0 \\ 1 & 0 & 0 & 0\end{array}\right\|,\left\|\begin{array}{cccc}0 & 0 & p c & 0 \\ 1 & 0 & 0 & 0\end{array}\right\|$ and $\left\|\begin{array}{lllc}0 & 0 & 0 & p d \\ 1 & 0 & 0 & 0\end{array}\right\|$, respectively, in $\mathrm{SL}_{n}(\mathbb{Z})$

There is a surjection $\phi: \Gamma_{p} \rightarrow M_{n}\left(\mathbb{Z}_{p}\right) \cong \mathbb{Z}_{p}^{n^{2}}$ of the congruence subgroup to an additive matrix group given by $\phi(A)=\left[\frac{1}{p}(A-I)\right]_{p}$. In other words, since $A \in \Gamma_{p}$, it is of the form $A=I+p C$, for some unique square matrix $C$. Then we define $\phi(A)=C$. Indeed, this map $\phi$ is a homomorphism because $\phi\left(A A^{\prime}\right)=\phi((1+p C)(1+$ $\left.\left.p C^{\prime}\right)\right)=\phi\left(I+p\left(C+C^{\prime}\right)+O\left(p^{2}\right)\right)=\left[C+C^{\prime}+O(p)\right]_{p}=\left[C+C^{\prime}\right]_{p}=\phi(A)+\phi\left(A^{\prime}\right)$. Because of cross terms, the induced map $\phi_{*}$ is nontrivial in $\bmod p$ homology.

Proposition 2.1. The group $H_{3}\left(\Gamma_{p} ; \mathbb{Z}\right)$ contains a nontrivial element of finite order $p$.

Proof. The element $\langle p b\rangle$ is nonzero in $H_{1}\left(\right.$ Heis $\left._{p} ; \mathbb{Z}\right)$ because its image under the $\operatorname{map} \phi_{*}: H_{1}\left(\Gamma_{p} ; \mathbb{Z}\right) \rightarrow H_{1}\left(\mathbb{Z}_{p}^{n^{2}} ; \mathbb{Z}\right)$ induced by $\phi$ is nonzero. But $p\langle p b\rangle$ is zero in $H_{1}\left(\right.$ Heis $\left._{p} ; \mathbb{Z}\right)$, so $\langle p b\rangle$ is 0 in $H_{1}\left(\right.$ Heis $\left._{p} ; \mathbb{Q}\right)$. In other words, this copy of $\mathbb{Z}$ is not integrally zero but is rationally zero. It is clear that $\operatorname{Heis}_{p}^{+} \cong \operatorname{Heis}_{p} \times(p \mathbb{Z})^{2}$. Then $\langle p b\rangle \times\langle p c\rangle \times\langle p d\rangle$ is integrally nonzero but is 0 in $H_{3}\left(\operatorname{Heis}_{p}^{+} ; \mathbb{Q}\right)$. Indeed, the $\langle p b\rangle$ bounds some $Q$, and so $\langle p b\rangle \times\langle p c\rangle \times\langle p d\rangle$ bounds $\mathbb{T}^{2} \times Q$. It is therefore also rationally zero and so has finite order in $H_{3}\left(\Gamma_{p} ; \mathbb{Z}\right)$.

Proposition 2.2. The group $H^{4}\left(\Gamma_{p} ; \mathbb{Z}\right)$ is nontrivial.

Proof. We have a universal coefficient theorem for cohomology given by

$$
H^{4}\left(\Gamma_{p} ; \mathbb{Z}\right)=\operatorname{Hom}\left(H_{4}\left(\Gamma_{p} ; \mathbb{Z}\right), \mathbb{Z}\right) \oplus \operatorname{Ext}\left(H_{3}\left(\Gamma_{p} ; \mathbb{Z}\right), \mathbb{Z}\right)
$$


The previous proposition gives a nontrivial Ext term.

Therefore, to identify a non-equivalent manifold structure in $S^{T o p}\left(M_{p}\right)$, we start by studying the collection $N^{T o p}\left(B \Gamma_{p}\right)$ of normal invariants.

Proposition 2.3. For $p$ sufficiently large, there is a map $\nu: B \Gamma_{p} \rightarrow F / O$ so that $p_{1}[1 / 2](\nu)$ is nontrivial, where $p_{1}[1 / 2]$ is defined via the diagram as follows:

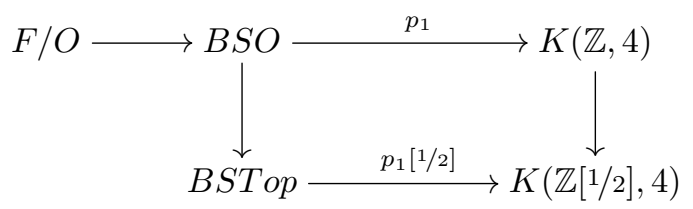

Remark 2.4. The fact that $H^{4}(B S T o p ; \mathbb{Z}[1 / 2]) \rightarrow H^{4}(B S O ; \mathbb{Z}[1 / 2])$ is an isomorphism follows from the calculations that $\pi_{i}(T o p / P L)=0$ for $i \neq 3$ and $\pi_{3}(T o p / P L)=$ $\mathbb{Z}_{2}$ and that $\pi_{i}(P L / O)=0$ for $i<7$. See Kirby-Siebenmann [33]. The lift of $p_{1} \in H^{4}(B S O ; \mathbb{Z})$ to $H^{4}(B S T o p ; \mathbb{Z}[1 / 2])$ will be called $p_{1}[1 / 2]$.

Proof. (of Proposition) We know that $B \Gamma_{p}$ is a finite complex of some finite dimension $k$, independent of $p$. If $p>k$ then $\pi_{i}(B F) \otimes \mathbb{Z}_{(p)}=0$ for all $i \leq k+1$. Assume that $p$ is sufficiently large so that $\pi_{i}(P L / O) \otimes \mathbb{Z}_{(p)}=0$ for all $i \leq k+1$. Then, upon localizing at $p$, we have the equivalences

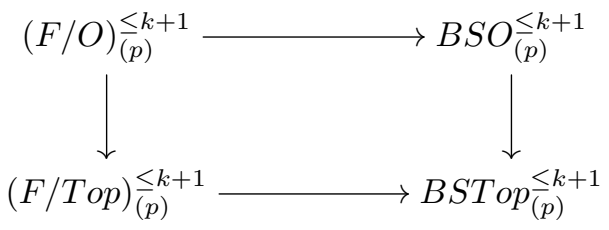

where the superscript $\leq k+1$ refers to the stage in the Postnikov decomposition. Note that Bott periodicity (formulated in terms of the Chern character inducing an isomorphism $\pi_{2 n}(B U) \rightarrow \mathbb{Z}$ ) implies that, for $p>k+1$, the map $\oplus p_{i}: B S O \leq k+1 \rightarrow$ $\prod K(\mathbb{Z}, 4 i)^{\leq k+1}$ is a $\mathbb{Z}_{(p)}$-equivalence. The result now follows.

As a consequence, for $p$ large, we can find a smooth normal invariant of a proper homotopy equivalence (by the $\pi$ - $\pi$ theorem) such that the underlying tangent bundles can be distinguished using $p_{1}[1 / 2]$, a topological invariant.

By Theorem 3 of Anderson-Hodgkin [1], there is no map $K(\mathbb{Z}, 4) \rightarrow F / T o p$ that is nontrivial on the finite skeleta. For this reason, we need to restrict ourselves to a finite complex mapping into $K(\mathbb{Z}, 4)$ and then split it (after multiplying by some appropriate constant).

Theorem 2.5. Let $n \geq 5$ and $G=\mathrm{SL}_{n}(\mathbb{R})$. Let $\Gamma_{p}$ be the principal $p$-congruence subgroup of $G$. Then for $p$ sufficiently large, the manifold $M=\Gamma_{p} \backslash \mathrm{SL}_{n}(\mathbb{R}) / \mathrm{SO}_{n}(\mathbb{R})$ is properly strongly nonrigid; i.e. there is a $N$ proper homotopy equivalent to $M$ that is not homeomorphic to $M$. 
Proof. Construct $\Gamma=\Gamma_{p}$ as above. Mostow's rigidity theorem implies that all self-equivalences of arithmetic manifolds come from isometries. In particular the Pontrjagin class $p_{1}$ is preserved under pullback. Therefore the constructed manifolds in the previous discussion are genuinely non-homeomorphic to the standard $\Gamma \backslash G / K$.

Remark 2.6. The Bass-Milnor-Serre Theorem states that $\mathrm{SL}_{n}(\mathbb{Z})$ satisfies the Congruence Subgroup Property with $n \geq 3$; i.e. given a subgroup $\Gamma \leq \mathrm{SL}_{n}(\mathbb{R})$ of finite index, we can find a principal congruence subgroup $\Gamma_{k} \leq \Gamma$ of finite index. Note that whenever $k \mid \ell$, we have $\Gamma_{\ell} \leq \Gamma_{k}$. Therefore we can find a sublattice $\Gamma_{\ell} \leq \Gamma$ of finite index such that $\ell$ is divisible by a sufficiently large prime. The results of this section can then be extended to lattices commensurable with $\mathrm{SL}_{n}(\mathbb{Z})$. Recall that two lattices $\Delta$ and $\Delta^{\prime}$ are commensurable if their intersection is of finite index in both of them.

The argument in the proof of Theorem 2.5 fails when $n=4$, but we will deal with this case in a later section.

\section{Proof FOR LARGe $\mathbb{Q}$-RANK}

Now we consider the case of general arithmetic lattices in a semisimple Lie group $G$. We rely on a theorem of Farb and Shalen [22] to prove the existence of an appropriate abelian sublattice isomorphic to $\mathbb{Z}^{3}$.

Theorem 3.1. (Farb-Shalen) Let $\Gamma$ be a lattice in a simple algebraic group $G$ over $\mathbb{Q}$. Let $d=\operatorname{rank}_{\mathbb{Q}}^{G}(\Gamma) \geq 6$ (can be reduced to 5 if we omit type $D_{5}$ ). Then $\Gamma$ contains two commuting subgroups $A$ and $B$ which are isomorphic to irreducible lattices with $\mathbb{Q}$-ranks 2 and $d-3$.

Proof. By the Margulis Arithmeticity Theorem, we can assume that $\Gamma$ is the $\mathbb{Z}$ points of such a group $G$. If $G$ is simple, then the Dynkin diagrams for the root system $\Phi$ of $G$ show that, when $d \geq 7$, one can remove a vertex of the diagram to obtain a graph with two components: one with two vertices and one with at least $d-3$ vertices. Let $G_{1}$ and $G_{2}$ be the root subgroups corresponding to these components of the Dynkin diagram. Then $G_{1}$ commutes with $G_{2}$. The group of $\mathbb{Q}$-points of $G_{1}$ has $\mathbb{Q}$-rank at least 2 , and the group of $\mathbb{Q}$-points of $G_{2}$ has $\mathbb{Q}$-rank at least $d-3$. Results by Borel and Harish-Chandra (see for example Zimmer [53]) show that $A=\Gamma \cap G_{1}$ and $B=\Gamma \cap G_{2}$ are arithmetic lattices in $G_{1}$ and $G_{2}$. Then $A$ and $B$ have the necessary properties.

Proposition 3.2. Let $\Gamma$ be a lattice in a semisimple algebraic group $G$ over $\mathbb{Q}$ with $\mathbb{Q}$-rank at least 6 . There is a nontrivial element in $H_{3}(\Gamma ; \mathbb{Z})$ which is rationally zero.

Proof. According to Farb-Shalen, we have two commuting subgroups represented by irreducible lattices $\Gamma_{1}$ and $\Gamma_{2}$ with $\mathbb{Q}$-ranks at least 2 , corresponding to two 
disjoint subgraphs of the Dynkin diagram of $G$. Since the rank of $\Gamma_{1}$ is 2 , we can identify an abelian sublattice in $\Gamma_{1}$ isomorphic to $\mathbb{Z}^{2}$. Since the other component is associated with a lattice of $\mathbb{Q}$-rank at least 2 , then $\Gamma_{2}$ has a finite abelianization by property $T$ (see the general discussion in [8]). Therefore there is a nonzero element in $H_{1}\left(\Gamma_{2} ; \mathbb{Z}\right)$ of finite order. The three-dimensional class in $H_{3}(\Gamma ; \mathbb{Z})$ that emerges from this computation is rationally trivial.

The techniques of the previous section give the following:

Theorem 3.3. Let $\Gamma$ be a lattice in a semisimple algebraic group $G$ over $\mathbb{Q}$ with $\mathbb{Q}$-rank at least 6. Let $K$ be a maximal compact subgroup of $G$. Then there is $\Gamma^{\prime} \leq \Gamma$ of finite index such that $\Gamma^{\prime} \backslash G / K$ is strongly nonrigid.

Proof. Let $\Gamma$ be the integer points of a rational algebraic group. We will consider, as in the previous section, a congruence subgroup associated to a large prime. As before, it has a homomorphism onto the Lie algebra of a finite field. Going deeper by powers of $p$, we obtain similar homomorphisms for these deeper subgroups as well. It is clear that this tower converges to the trivial group, so given a free abelian group in our lattice, one can find a level so that the intersection of that group with the relevant lattice has as its image a free abelian $p$-group with the same number of factors.

Theorem 3.4. If $\operatorname{rank}_{\mathbb{Q}}^{G} \Gamma \geq 5$ there is a nontrivial $N \in S^{T o p}(\Gamma \backslash G / K)$ which is smooth.

Proof. As before, the three-dimensional torsion homology class gives rise to an element in $H^{4}(-; \mathbb{Z})$. If it is $p$-torsion for a large enough prime (depending on dimension) we are guaranteed (as in Proposition 2.3) a lifting to $F / O$, giving us a smooth normal invariant detected by a topologically invariant Pontrjagin class.

It is meaningful to ask whether the elements constructed here become trivial upon passing to further covers. We believe that they do, and only know of very occasional elements constructed by finite-order methods that continue to be nontrivial upon passing to finite covers. We note here that the elements constructed in our previous paper [18], upon passing to appropriate finite covers, all become trivial.

Proposition 3.5. The elements constructed in [18] by strong approximation represent the zero element in $S^{\text {virt }}(\Gamma \backslash G / K)$; i.e. if $N=\Gamma \backslash G / K$ is an arithmetic space constructed by strong approximation and if $(P, f)$ is a nontrivial element in $S^{T o p}(N)$, then there is a finite lift $N^{\prime}=\Gamma^{\prime} \backslash G / K$ of $N$ for which the corresponding $\Gamma^{\prime}$-lift $\left(P^{\prime}, f^{\prime}\right)$ of $(P, f)$ is the trivial element in $S^{T o p}\left(N^{\prime}\right)$.

Proof. By the strong approximation of Lubotzky, there is a subgroup $\Gamma^{\prime} \leq \Gamma$ with an epimorphism $\phi: \Gamma^{\prime} \rightarrow \mathbb{Z}_{2}$. For each $k \geq 1$, we say that $\phi$ lifts to stage $k$ if there 
is a commutative diagram

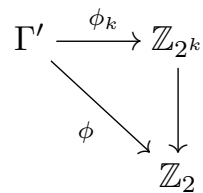

Our proof in [18] shows that there is a minimum $n$ for which $\phi$ does not lift to stage $n$. Consider then the lift to $\Gamma^{\prime} \rightarrow \mathbb{Z}_{2^{n-1}}$. This lift gives an element $u$ in $H^{1}\left(\Gamma^{\prime} ; \mathbb{Z}_{2^{n-1}}\right)$ whose Bockstein element (associated to $0 \rightarrow \mathbb{Z}_{2} \rightarrow \mathbb{Z}_{2^{n}} \rightarrow \mathbb{Z}_{2^{n-1}} \rightarrow$ $0)$ is nontrivial in $H^{2}\left(\Gamma^{\prime} ; \mathbb{Z}_{2}\right)$. Otherwise it would lift nontrivially higher. In other words, if $\beta_{\Gamma^{\prime}}: H^{1}\left(\Gamma^{\prime} ; \mathbb{Z}_{2^{n-1}}\right) \rightarrow H^{2}\left(\Gamma^{\prime} ; \mathbb{Z}_{2}\right)$ is the Bockstein map, then $\beta_{\Gamma^{\prime}}(u) \neq 0$.

Now let $\Delta$ be the kernel of the map $\phi_{n-1}: \Gamma^{\prime} \rightarrow \mathbb{Z}_{2^{n-1}}$. Since the restriction map $\left.\phi_{n-1}\right|_{\Delta}: \Delta \rightarrow \mathbb{Z}_{2^{n-1}}$ is zero, it lifts further to $\mathbb{Z}_{2^{n}}$. If $v$ represents the map $\left.\phi_{n-1}\right|_{\Delta}$ in $H^{1}\left(\Delta ; \mathbb{Z}_{2^{n-1}}\right)$ and the Bockstein map is given by $\beta_{\Delta}: H^{1}\left(\Delta ; \mathbb{Z}_{2^{n-1}}\right) \rightarrow H^{2}\left(\Delta ; \mathbb{Z}_{2}\right)$, then the commutative diagram

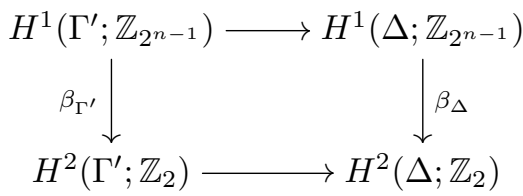

gives $\beta_{\Delta}(v)=0$ by naturality.

\section{The Hermitian symmetric Case}

In this section we completely classify properly nonrigid Hermitian locally symmetric manifolds in high real dimension. Hermitian locally symmetric manifolds are Riemmanian symmetric spaces with a parallel complex structure with respect to which the Riemannian metric is Hermitian. In terms of the classification of Riemannian symmetric spaces, the Hermitian symmetric spaces are the four infinite series AIII, BDI with $p=2$ or $q=2$, DIII and CI, and two exceptional spaces, namely EIII and EVII. See Helgason [29] for additional information on Hermitian symmetric spaces.

\begin{tabular}{llc} 
& Manifold & Complex dimension \\
\hline AIII & $\mathrm{SL}(p+q, \mathbb{C})$ & $p q$ \\
BDI $(q=2)$ & $\mathrm{SL}(p+2, \mathbb{C})$ & $p$ \\
DIII & $\mathrm{SL}(2 n, \mathbb{C})$ & $\frac{1}{2} n(n-1)$ \\
CI & $\mathrm{Sp}(2 n, \mathbb{C})$ & $\frac{1}{2} n(n+1)$ \\
EIII & $E_{6}^{\mathbb{C}}$ & 16 \\
EVII & $E_{7}^{\mathbb{C}}$ & 27
\end{tabular}

In the following theorem, we demonstrate that a Hermitian locally symmetric manifold, when it is properly rigid, is properly rigid for fairly simple reasons.

Theorem 4.1. If $M=\Gamma \backslash G / K$ is a Hermitian locally symmetric manifold of real dimension at least 5 , then the following are equivalent: 
(1) The $\mathbb{Q}$-rank of $\Gamma$ is at least 3.

(2) The space $M$ is properly topologically nonrigid.

If these conditions are satisfied, then infinitely many elements in $S^{T o p}(M)$ are in fact detected by a rational topological version of the first Pontrjagin class in $H^{4}(M ; \mathbb{Q})$. Also in this case, the space $M$ is properly nonrigid among smooth manifolds (where the conclusion is still homeomorphism).

Proof. Suppose that $\operatorname{rank}_{\mathbb{Q}}(\Gamma) \leq 2$. Then the topological rigidity of $M=\Gamma \backslash G / K$ can be inferred from the work of Farrell-Jones [24] and Bartels-Lück-Reich-Rüping [7] by the following argument. In Block-Weinberger [9], Theorem 1.3 shows that proper rigidity of the relevant assembly map depends on an isomorphism statement for the manifold at infinity. By Borel-Serre [10] there is an exact sequence $1 \rightarrow$ $F_{\infty} \rightarrow \pi_{1}(\partial M) \rightarrow \Gamma \rightarrow 1$, where $F_{\infty}$ is the free group of countably infinitely many generators and $\partial M$ is the Borel-Serre boundary. We identify $H_{*}\left(B \pi_{1}(\partial M), \mathbb{L}(e)\right)$ with $H_{*}\left(B \Gamma ; \mathbb{H}\left(B F_{\infty} ; \mathbb{L}(e)\right)\right)$ and then further identify this group with $H_{*}\left(B \Gamma ; \mathbb{L}\left(F_{\infty}\right)\right)$ by the Borel conjecture for free groups (infinite rank makes no difference by a limit argument). Finally the isomorphism $H_{*}\left(B \Gamma ; \mathbb{L}\left(F_{\infty}\right)\right) \cong L_{*}\left(\pi_{1}(\partial M)\right)$ is exactly the conclusion of the Farrell-Jones isomorphism conjecture in the nontrivial coefficient system $\mathbb{Z} F_{\infty}$.

If $\operatorname{rank}_{\mathbb{Q}}(\Gamma) \geq 3$, then we are in the $\pi-\pi$ situation, so it suffices to prove that $H^{4}(\Gamma \backslash G / K ; \mathbb{Z})$ is nontrivial. When $X=\Gamma \backslash G / K$ is a Hermitian symmetric space, one can take its Baily-Borel compactification $\bar{X}$, constructed by adding to $X$ a singular set $\Sigma$, to obtain a singular projective variety. It has a nontrivial evendimensional fundamental class $[\bar{X}] \in H_{d}(\bar{X} ; \mathbb{Z})$ (see Baily-Borel [5] and Borel-Ji [14]). In fact, by taking hyperplane sections, one can find nontrivial elements in homological dimensions $d-2 k$ for all $k$. It is known that the complex codimension of $\Sigma \hookrightarrow \bar{X}$ in our case is at least 3 (see Jost-Yau [32]), in which case there is an isomorphism $H_{*}(\bar{X} ; \mathbb{Z}) \hookrightarrow H_{*}(\bar{X}, \Sigma ; \mathbb{Z})$ above the dimension of $\Sigma$. In particular we have an isomorphism if $*=d-4$. By excision, the above group is isomorphic to $H_{*}\left(M_{\Sigma}, \partial M_{\Sigma} ; \mathbb{Z}\right)$, where $M_{\Sigma}$ is the complement of a tubular neighborhood of $\Sigma$ and $\partial M_{\Sigma}$ is its boundary. The boundary is in fact the Borel-Serre boundary and $M_{\Sigma}$ is a manifold. We then have $H_{d-4}(\bar{X} ; \mathbb{Z}) \cong H_{d-4}\left(M_{\Sigma}, \partial M_{\Sigma} ; \mathbb{Z}\right) \cong H^{4}\left(M_{\Sigma} ; \mathbb{Z}\right) \cong$ $H^{4}(\Gamma \backslash G / K ; \mathbb{Z})$, so that $H^{4}(\Gamma \backslash G / K ; \mathbb{Q})$ is nontrivial. Of course this result implies that $\Gamma \backslash G / K$ is properly nonrigid and its topological structure set is infinite. Moreover, as in Proposition 2.3 and Theorem 3.4, infinitely many of these elements are smoothable.

In addition, when (1) and (2) are satisfied, there are infinitely exotic versions $N$ of $M$ such that, for all $\Delta \leq \Gamma$ of finite index, the lifts $N_{\Delta}$ and $M_{\Delta}$ are (still) not homeomorphic.

Remark 4.2. Now that we know that the structure set is infinite for a reason based on $p_{1}$, we can actually realize a subset containing a lattice in $H^{4}(\Gamma ; \mathbb{Z}) /$ tors coming from smooth exotic versions of $\Gamma \backslash G / K$. It is easy to see that the automorphisms of $\Gamma$, lying in $\mathrm{SL}\left(H^{4}(\Gamma ; \mathbb{Z}) /\right.$ tors $)$, must have infinitely many orbits in our set, and 
therefore we produce infinitely many manifolds, not just proper homotopy equivalences.

\section{The VIRTUAL STRUCTURE SET}

Having examined the proper rigidity or nonrigidity of locally symmetric spaces in a number of different cases, we now wish to define a notion of a virtual structure set associated to the covers of a manifold of the form $\Gamma \backslash G / K$. Suppose that $\Gamma$ and $\Gamma^{\prime}$ are both torsion-free lattices of $G$ with $\Gamma^{\prime} \leq \Gamma$. Suppose that the index $\left[\Gamma: \Gamma^{\prime}\right]$ is finite. There is an induced covering map $\Gamma^{\prime} \backslash G / K \rightarrow \Gamma \backslash G / K$, called a lift of $\Gamma \backslash G / K$, which induces a map $S^{T o p}\left(\Gamma^{\prime} \backslash G / K\right) \rightarrow S^{T o p}(\Gamma \backslash G / K)$ of topological structure sets. There is then a transfer map $S^{T o p}(\Gamma \backslash G / K) \rightarrow S^{T o p}\left(\Gamma^{\prime} \backslash G / K\right)$. Suppose $\Lambda$ denotes the inverse system of inclusions $\Lambda: \cdots \rightarrow \Gamma_{3} \rightarrow \Gamma_{2} \rightarrow \Gamma_{1}=\Gamma$ with each $\Gamma_{i} \leq G$ torsion-free and each index $\left[\Gamma_{i}: \Gamma_{i+1}\right]$ finite. We will say that $\Lambda$ is a finite index sequence of torsion-free lattices in $G$. In this case, the induced direct system $S^{T o p}(\Gamma \backslash G / K)=S^{T o p}\left(\Gamma_{1} \backslash G / K\right) \rightarrow S^{T o p}\left(\Gamma_{2} \backslash G / K\right) \rightarrow S^{T o p}\left(\Gamma_{3} \backslash G / K\right) \rightarrow \cdots$ of transfer maps gives rise to a limit object $S_{\Lambda}^{\text {virt }}(\Gamma \backslash G / K) \equiv \lim S^{T o p}\left(\Gamma_{i} \backslash G / K\right)$, called the virtual structure set of $\Gamma \backslash G / K$ with respect to $\Lambda$. Note that $S_{\Lambda}^{\text {virt }}(\Gamma \backslash G / K)$ has a group structure because each $S^{T o p}\left(\Gamma_{i} \backslash G / K\right)$ can be given a group structure for which all the transfer maps are homomorphisms.

There are several natural examples of sequences $\Lambda$ that can be studied.

(1) Let $\Delta_{k}$ be the intersection of all subgroups of $\Gamma$ of index at most $k$. Define by $S^{\text {virt }}(\Gamma \backslash G / K)$ the structure set associated to the particular sequence $\Lambda: \cdots \rightarrow \Delta_{3} \rightarrow \Delta_{2} \rightarrow \Delta_{1} \rightarrow \Gamma$. If $\Lambda^{\prime}: \cdots \rightarrow \Gamma_{3} \rightarrow \Gamma_{2} \rightarrow \Gamma_{1} \rightarrow \Gamma$ is a finite index sequence of torsion-free lattices of $G$, then there is an increasing sequence $n_{i}$ of positive integers with maps $\Gamma_{i} \rightarrow \Delta_{n_{i}}$ such that all relevant compositions commute. Therefore $\Lambda$ can be regarded as the terminal object in the collection of all finite index sequences of torsion-free lattices in $G$. We then suppress the mention of $\Lambda$ in the notation $S^{\text {virt }}(\Gamma \backslash G / K)$, which we then call the universal virtual structure set. Our attention will be mainly be spent on the determination of this set. In the arithmetic case, this set is an invariant of $G(\mathbb{Q})$.

(2) Let $\Gamma$ be a lattice in a linear algebraic group. We can then embed $\Gamma$ as a subgroup of $\mathrm{SL}_{N}(\mathbb{Z})$ for large $N$. In $\mathrm{SL}_{N}(\mathbb{Z})$ we have the sequence of principal $m$-congruence subgroups given by $\Delta(m)=\operatorname{ker}\left(\mathrm{SL}_{N}(\mathbb{Z}) \rightarrow \mathrm{SL}_{N}\left(\mathbb{Z}_{m}\right)\right)$, and we can then consider the corresponding congruence subgroups of $\Gamma$ given by $\Gamma_{m}=\Gamma \cap \Delta(m)$. An interesting sequence is then given by $\Lambda: \cdots \rightarrow \Gamma_{m_{3}} \rightarrow \Gamma_{m_{2}} \rightarrow \Gamma_{m_{1}}$, where, for all $i$, there is a prime $p_{i}$ for which $m_{i}=p_{i} m_{i+1}$.

(3) Using the notation in (2), fix a positive prime $p \in \mathbb{Z}$ and consider the sequence $\Lambda_{p}: \cdots \rightarrow \Gamma_{p^{3}} \rightarrow \Gamma_{p^{2}} \rightarrow \Gamma_{p}$. 
Example 5.1. Let $\Lambda$ as in (2) above. When the $\mathbb{Q}$-rank of $\Gamma$ is large, then $S_{\Lambda}^{\text {virt }}(\Gamma \backslash G / K)$ contains an infinitely generated torsion group (as we showed in Section 3 ).

Example 5.2. Let $\Pi$ be a product of a product of three punctured tori. We mentioned in the introduction that the universal virtual structure set $S^{\text {virt }}(\Pi)$ vanishes. However, using the notation in (3) above, we note that the virtual structure set $S_{\Lambda_{2}}^{v i r t}(\Pi)$ is trivial while, when $p$ is an odd prime, the structure set $S_{\Lambda_{p}}^{\text {virt }}(\Pi)$ is an infinite sum of copies of $\mathbb{Z}_{2}$.

At this stage we turn to a discussion of the final goal of the paper: the determination of the universal virtual structure set $S^{\text {virt }}(\Gamma \backslash G / K)$ in a variety of cases. We begin with some terminology and a theorem of Ash.

Notation 5.3. Suppose that $G$ is a semisimple algebraic group defined over $\mathbb{Q}$ with maximal compact subgroup $K \subseteq G(\mathbb{R})$ and $\Gamma_{0} \subseteq G(\mathbb{Q})$ a torsion-free arithmetic subgroup. Let $\Gamma$ be a subgroup of $\Gamma_{0}$ of finite index and let $e_{P}(\Gamma)$ be the number of distinct double cosets $\left(P \cap \Gamma_{0}\right) \backslash \Gamma_{0} / \Gamma$ for each fixed $\mathbb{Q}$-parabolic subgroup $P$ of $G$ and let $d$ be the real dimension of the $\mathbb{R}$-unipotent radical of such a $P$.

Our main tool in computing the virtual structure set is the following theorem of Ash.

Theorem 5.4. (Ash [3] and Schwermer [45]) In the situation given above, we have

$$
\operatorname{dim} H^{d}(\Gamma ; \mathbb{R}) / H_{(2)}^{d}(\Gamma ; \mathbb{R}) \geq e_{P}(\Gamma) .
$$

In addition, the term $e_{P}(\Gamma)$ increases as $\Gamma$ decreases, so the number of linearly independent non-square-integrable cohomology classes will tend to infinity.

We note that, if $X$ is a space and $Y$ is a finite-sheeted cover of $X$, then the rational cohomology of $X$ injects into the rational cohomology of $Y$, so any nonzero elements in the former persist into the latter. Our discussion in Section 1 indicates that, using the result of Ash above, the virtual structure set of an arithmetic manifold $\Gamma \backslash G / K$ will be infinite if we can find a $\mathbb{Q}$-parabolic whose unipotent radical has real dimension $d$ divisible by 4 . Our analysis involves a calculation for each Lie group via its Dynkin diagram. These computations are relegated to Section 6 of the paper. In the following lemma and thereafter, the term "horospherical" refers to a unipotent radical of a parabolic subgroup.

Lemma 5.5. Let $G$ be a connected, semisimple algebraic group over $\mathbb{Q}$. If $\operatorname{rank}_{\mathbb{Q}} G \geq$ 4 , then $G$ has a nontrivial horospherical $\mathbb{Q}$-subgroup $U$ such that $\operatorname{dim}_{\mathbb{R}} U$ is divisible by 4 .

Proof. Suppose $G$ does not have such a horospherical $\mathbb{Q}$-subgroup. Write $G=$ $G_{1} \times \cdots \times G_{k}$ with $k \geq 2$, where each $G_{i}$ is almost simple. We may assume that each $G_{i}$ is isotropic (since $\mathbb{Q}$-anisotropic factors do not affect the $\mathbb{Q}$-rank).

Case 1. Assume that $k=1$. Inspection of the calculations verifies that every almost-simple group of $\mathbb{Q}$-rank $\geq 4$ has an appropriate horospherical $\mathbb{Q}$-subgroup. More precisely, the only almost-simple $\mathbb{Q}$-groups $G$ of $\mathbb{Q}$-rank $\geq 3$ for which there 
does not exist a nontrivial horospherical $\mathbb{Q}$-subgroup whose dimension is divisible by 4 are:

(1) type $C_{n}$ or ${ }^{1,2} D_{n}$ of $\mathbb{Q}$-rank 3 , with $d=2$ (i.e. constructed from a quaternion division algebra), or

(2) type $E_{7}$ of $\mathbb{Q}$-rank 3 .

Case 2. Assume that $k=2$. From Case 1, we know that $\operatorname{rank}_{\mathbb{Q}} G_{i} \leq 3$ for all $i$. Suppose that $\operatorname{rank}_{\mathbb{Q}} G_{1}=3$. Let $m$ be the dimension of a nontrivial horospherical $\mathbb{Q}$-subgroup $U_{2}$ of $G_{2}$. Then $m \not \equiv 0 \bmod 4$. Also, we know that $G_{1}$ is one of the groups listed in Case 1, so, by inspection of the calculations, we see that it has a horospherical subgroup $U_{1}$ such that $\operatorname{dim}_{\mathbb{R}} U_{1} \equiv-\operatorname{dim}_{\mathbb{R}} U_{2} \bmod 4$. Then $\operatorname{dim}\left(U_{1} \times U_{2}\right)$ is divisible by 4 .

We may now assume $\operatorname{rank}_{\mathbb{Q}} G_{1}=\operatorname{rank}_{\mathbb{Q}} G_{2}=2$. As was mentioned above, inspection of the calculations establishes that there is a nontrivial horospherical subgroup $U_{i}$ of $G_{i}$ such that $\operatorname{dim}_{\mathbb{R}} U_{i}$ is even. Then either $\operatorname{dim}_{\mathbb{R}} U_{1}$ or $\operatorname{dim}_{\mathbb{R}} U_{2}$ or $\operatorname{dim}\left(U_{1} \times U_{2}\right)$ is divisible by 4 .

Case 3. Assume that $k=3$. There must exist $k \in\{1,3\}$ such that, for each $i$, the dimension of every nontrivial horospherical $\mathbb{Q}$-subgroup of $G_{i}$ is congruent to $k$ mod 4. Since groups of rank at least 2 have a horospherical subgroup of even dimension, we have $\operatorname{rank}_{\mathbb{Q}} G_{i}=1$. Therefore $\operatorname{rank}_{\mathbb{Q}} G=k=3$, contradicting the fact that $\operatorname{rank}_{\mathbb{Q}} G \geq 4$.

Case 4. Assume that $k \geq 4$. Every collection of at least four integers has a nonempty subset whose sum is divisible by 4 .

Remark 5.6. The calculations show that any group of rank at least 2 has a nontrivial horospherical $\mathbb{Q}$-subgroup of even dimension. Therefore, if $G=\operatorname{Res}_{\mathbb{F} / \mathbb{Q}} H$ is a restriction of scalars with $\operatorname{rank}_{\mathbb{Q}} G \geq 2$, and $G$ does not have a horospherical $\mathbb{Q}$ subgroup of dimension divisible by 4 , then $\mathbb{F}$ must have odd degree over $\mathbb{Q}$.

The lemma directly proves the following theorem about the virtual structure set.

Theorem 5.7. Let $G$ be a connected, semisimple algebraic group over $\mathbb{Q}$ and $\Gamma$ an arithmetic lattice in $G$. If $\operatorname{rank}_{\mathbb{Q}} G \geq 4$, then $S^{\text {virt }}(\Gamma \backslash G / K) \otimes \mathbb{Q}$ has infinite $\mathbb{Q}$-dimension. If $\operatorname{rank}_{\mathbb{Q}} G \geq 3$ and $\Gamma$ is irreducible, then the same result holds as long as $G$ is not of the types listed in Case 1 of the previous lemma. If $\Gamma=\mathrm{SL}_{n}(\mathcal{O})$ for a number ring $\mathcal{O}$, or if $\Gamma$ is the result of restriction by scalars for an extension $\mathbb{Q} \rightarrow \mathbb{F}$, where $[\mathbb{F}: \mathbb{Q}]$ is even, then the conclusion holds.

Remark 5.8. Let $T$ be the punctured torus, which has $\mathbb{Q}$-rank 1 . The triple product $T \times T \times T$ has $\mathbb{Q}$-rank 3 (e.g. using coarse volume growth) but the virtual structure set is trivial. So without the irreducibility criterion, this theorem is false. However it may be the only example.

One may ask, in the case when Ash's theorem can be applied to prove that $S^{\text {virt }}(M) \otimes$ $\mathbb{Q}$ has infinite as a $\mathbb{Q}$-vector space, how quickly the rank grows as a function of $\Gamma$. In other words, suppose that $G=\Gamma \backslash G / K$ has $\mathbb{Q}$-rank $\geq 4$. Using the notation 
of (1) in Section 5 , we let $\Delta_{k}$ be the intersection of all subgroups of $\Gamma$ of index at most $k$. Let $a_{k}(M)$ the rank of $S^{T o p}\left(\Delta_{k} \backslash G / K\right)$ as a vector space over $\mathbb{Q}$. It is a consequence of the paper of Ash [2] that in fact the quantity $a_{k}(M)$ grows as a power of the index $\left[\Gamma: \Delta_{k}\right]$ (or volume). One may then try to identify the manifolds $M=\Gamma \backslash G / K$ for which the growth rate is linear.

Proposition 5.9. Let $M=\Gamma \backslash G / K$ have $\mathbb{Q}$-rank at least 3, and let $M^{u}$ be the compact dual of $M$. Then $a_{k}(M)$ grows linearly in the index $\left[\Gamma: \Delta_{k}\right]$ iff the Euler characteristic $\chi\left(M^{u}\right)$ is nonzero and $\operatorname{dim} M \equiv 0 \bmod 8$.

Proof. This theorem is a consequence of Lück's approximation theorem relating $L^{2}$ cohomology of universal covers of compact spaces and the ordinary cohomology of their finite covers, together with the fact (first established by Cheeger and Gromov [20], but proved more conceptually by Gaboriau [26]) that, for nonuniform lattices, the $L^{2}$-cohomology of the universal covers of their Borel-Serre compactification is the same as that of the symmetric space. The details are as follows. The only nontrivial $L^{2}$-cohomology group arises in the middle dimension (see e.g. Olbrich [39]). Therefore the Betti numbers other than the middle one grow sublinearly. Consequently, linear growth occurs in a dimension that is a multiple of 4 iff the middle dimension is a multiple of four; i.e. $\operatorname{dim}(G / K)$ is a multiple of 8 , and the Euler characteristic is nonzero. However, by Gauss-Bonnet, the Euler characteristic of $M$ and of the compact dual are proportional (via the ratios of their volumes), completing the proof.

\section{Computations}

There is a method by which one can easily calculate the real dimension of the unipotent radical of a $\mathbb{Q}$-parabolic. The dimension of the unipotent radical associated to the Borel subgroup is the number of positive roots in the root system. For a smaller parabolic, we just need to subtract the number of positive roots that are in the Levi subgroup of the parabolic. The diagrams in Tits' Classification of algebraic semisimple groups [47] can be used to identify the Levi subgroup of each parabolic subgroup. The table on page 66 of Humphreys [30] tells us the number of positive roots there are in each simple factor of the Levi subgroup.

The complete list of Dynkin diagrams of semisimple groups over algebraically closed fields is given below. Each diagram determines a strict isogeny class of semisimple groups over any given field $k$. 


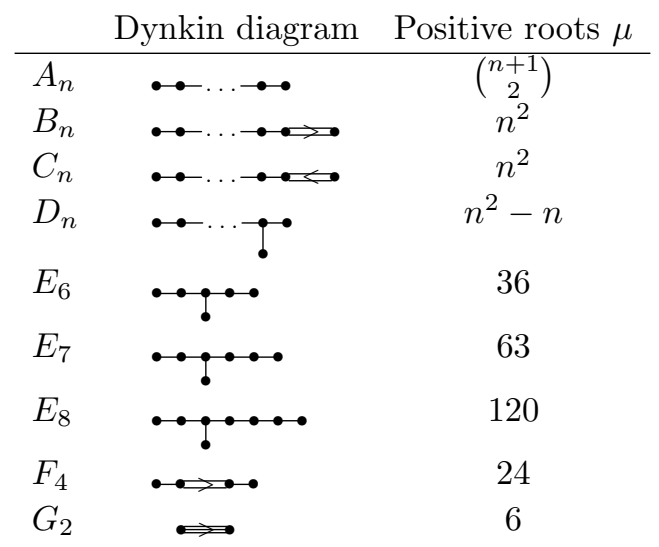

There is a natural one-to-one correspondence between the conjugacy classes of parabolic subgroups of $G$ and the subsets of the set $\Delta$ of all simple roots. In the following, we denote by $n$ the absolute (real) rank and $r$ the relative (rational) rank of the considered group, i.e. the total number of vertices and the number of distinguished orbits of the diagram. Note that $0 \leq r \leq n$. See Platonov-Rapinchuk [41] for a general treatment.

In the following pages, we examine each type of Lie group $G$ and calculate the real rank of the unipotent radical for various classes parabolic subgroup. We enumerate the Lie groups according to the subtypes given by Tits: ${ }^{1} A_{n},{ }^{2} A_{n}, B_{n}, C_{n},{ }^{1} D_{n}$, ${ }^{2} D_{n},{ }^{3,6} D_{4},{ }^{1} E_{6},{ }^{2} E_{6}, E_{7}, E_{8}, F_{4}, G_{2}$. The reader should refer to [47] for the subscripting and superscripting in this notation. For each of these types we use the Satake-Tits diagrams which classify real forms of the complex Lie algebra corresponding to the Dynkin diagrams. Our list of such diagrams is taken from Helgason [29], Satake [44], Onishchik-Vinberg [40] and Tits [47]. The nodes colored black correspond to the simple roots of the anisotropic kernel. We list the possible parabolic subgroups according to rational rank, which is given by the number of open circles (i.e. non-black nodes) in each diagram. A parabolic $\mathbb{Q}$-subgroup is determined by choosing any subset of these circles. Since we only want proper parabolics, our calculations always involve a nonempty subset. We then compute the real dimension of the associated unipotent radical by calculating the positive roots of the original diagram and then subtracting the positive roots of the subdiagrams that result upon deleting the open nodes.

Recall that, by Theorem 5.4 and Section 1, it suffices to find a parabolic whose unipotent radical has real dimension divisible by 4 . In the case of $\mathbb{Q}$-rank at least 4 , if we find one whose dimension is divisible by 4 , then we calculate no further. Otherwise we consider cases of larger parabolics by filling in various open nodes and calculating the real dimension of unipotent radical corresponding to the new Satake-Tits diagram. The number of positive roots of particular Lie group $G$ is denoted by $\mu$. 
The calculations below are done only for absolutely simple groups over $\mathbb{Q}$. For a restriction of scalars, the answers are to be multiplied by the degree of the field extension.

Type ${ }^{1} A_{n}$ : Here we have the special linear group $\mathrm{SL}_{r+1}(D)$, where $D$ is a central division algebra of degree $d$ over $k$. These constants satisfy $d(r+1)=n+1$ and $d \geq 1$.

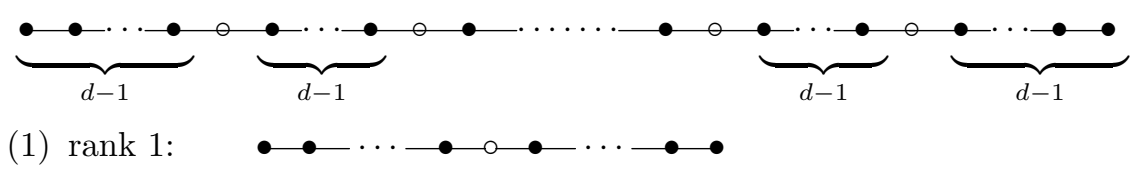

$$
\begin{aligned}
\operatorname{dim}_{\mathbb{R}} U & =\mu\left(A_{n}\right)-2 \mu\left(A_{d-1}\right) \\
& =\left(\begin{array}{c}
n+1 \\
2
\end{array}\right)-2\left(\begin{array}{l}
d \\
2
\end{array}\right) \\
& =\left(\begin{array}{c}
2 d \\
2
\end{array}\right)-2\left(\begin{array}{l}
d \\
2
\end{array}\right) \\
& =d^{2} \equiv \begin{cases}1 \bmod 4 & \text { if } d \text { odd }, \\
0 \bmod 4 & \text { if } d \text { even. }\end{cases}
\end{aligned}
$$

(2) $\operatorname{rank} 2$ :

$$
\begin{aligned}
\operatorname{dim}_{\mathbb{R}} U & =\mu\left(A_{n}\right)-3 \mu\left(A_{d-1}\right) \\
& =\left(\begin{array}{c}
n+1 \\
2
\end{array}\right)-3\left(\begin{array}{l}
d \\
2
\end{array}\right) \\
& =\left(\begin{array}{c}
3 d \\
2
\end{array}\right)-\left(\begin{array}{l}
d \\
2
\end{array}\right)-\left(\begin{array}{c}
2 d \\
2
\end{array}\right) \\
& =2 d^{2} \equiv \begin{cases}2 \bmod 4 & \text { if } d \text { odd } \\
0 \bmod 4 & \text { if } d \text { even }\end{cases}
\end{aligned}
$$

If we color in one of the two white circles, we have

$$
\begin{aligned}
\operatorname{dim}_{\mathbb{R}} U & =\mu\left(A_{n}\right)-\mu\left(A_{d-1}\right)-\mu\left(A_{2 d-1}\right) \\
& =\left(\begin{array}{c}
n+1 \\
2
\end{array}\right)-\left(\begin{array}{l}
d \\
2
\end{array}\right)-\left(\begin{array}{c}
2 d \\
2
\end{array}\right) \\
& =\left(\begin{array}{c}
3 d \\
2
\end{array}\right)-3\left(\begin{array}{l}
d \\
2
\end{array}\right) \\
& =3 d^{2} \equiv \begin{cases}3 \bmod 4 & \text { if } d \text { odd } \\
0 & \bmod 4 \text { if } d \text { even } .\end{cases}
\end{aligned}
$$

(3) rank $r \geq 3: \quad \circ \circ \cdots \circ$ if $d=1$ and $\bullet \circ \bullet \circ \bullet \cdots-\circ \bullet$ if $d=2$ 


$$
\begin{aligned}
\operatorname{dim}_{\mathbb{R}} U & =\mu\left(A_{n}\right)-\mu\left(A_{(k+1) d-1}\right)-(r-k) \mu\left(A_{d-1}\right) \\
& =\left(\begin{array}{c}
n+1 \\
2
\end{array}\right)-\left(\begin{array}{c}
(k+1) d \\
2
\end{array}\right)-(r-k)\left(\begin{array}{l}
d \\
2
\end{array}\right) \\
& =\left(\begin{array}{c}
d(r+1) \\
2
\end{array}\right)-\left(\begin{array}{c}
(k+1) d \\
2
\end{array}\right)-(r-k)\left(\begin{array}{l}
d \\
2
\end{array}\right) \\
& =\frac{d^{2}}{2}\left(r^{2}+r-k(k+1)\right) \\
& =\frac{d^{2}}{2}(r+k+1)(r-k) .
\end{aligned}
$$

This quantity is $0 \bmod 4$ when $r \equiv k \bmod 8$ or $r \equiv(7-k) \bmod 8$. For each $r$, one can choose an appropriate $k$ so that $\operatorname{dim}_{\mathbb{R}} U \equiv 0 \bmod 8$.

Type ${ }^{2} A_{n}$ : Here we have the special unitary group $\mathrm{SU}_{(n+1) / d}(D, h)$, where $D$ is a central division algebra of degree $d$ over a quadratic extension $k^{\prime}$ of $k$ with an involution of the second kind $\sigma$ such that $k=\left\{x \in k^{\prime}: x^{\sigma}=x\right\}$ and $h$ is a nondegenerate Hermitian form of index $r$ relative to $\sigma$. The constants satisfy $d \mid(n+$ 1) and $d \geq 1$. Also $2 r d \leq n+1$.

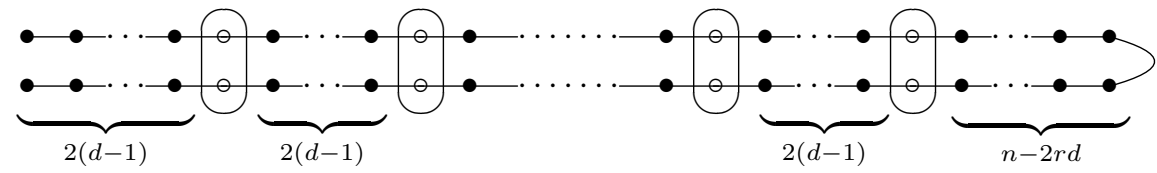

or

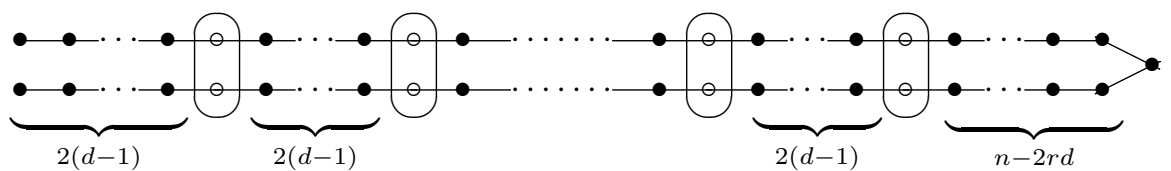

When $n+1=2 r d$, the right end of the second picture is replaced with an open circle.

(1) rank 1: $\bullet \bullet \cdots$ for $d=1$ and $\bullet \circ \bullet \bullet \cdots$ for $d=2$

$$
\begin{aligned}
\operatorname{dim}_{\mathbb{R}} U & =\mu\left(A_{n}\right)-2 \mu\left(A_{d-1}\right)-\mu\left(A_{n-2 d}\right) \\
& =\left(\begin{array}{c}
n+1 \\
2
\end{array}\right)-2\left(\begin{array}{l}
d \\
2
\end{array}\right)-\left(\begin{array}{c}
n-2 d+1 \\
2
\end{array}\right) \\
& =2 d n+2 d-3 d^{2} \\
& = \begin{cases}0 & \text { if } d \text { even, } \\
1 & \text { if } d \text { and } n \text { odd }, \\
3 & \text { if } d \text { odd and } n \text { even. }\end{cases}
\end{aligned}
$$

(2) $\operatorname{rank} r \geq 2$ :

$$
\circ \circ \bullet \bullet \cdots \text { for } d=1 \text { and } \bullet \circ \bullet \circ \bullet \bullet \bullet \cdots \text { for } d=2
$$




$$
\begin{aligned}
\operatorname{dim}_{\mathbb{R}} U & =\mu\left(A_{n}\right)-2 r \mu\left(A_{d-1}\right)-\mu\left(A_{n-2 r d}\right) \\
& =\left(\begin{array}{c}
n+1 \\
2
\end{array}\right)-2 r\left(\begin{array}{l}
d \\
2
\end{array}\right)-\left(\begin{array}{c}
n-2 r d+1 \\
2
\end{array}\right) \\
& = \begin{cases}r(2 n-2 r+1) & \text { if } d=1, \\
4 r n-8 r^{2} & \text { if } d=2 .\end{cases}
\end{aligned}
$$

When $d=2$ we have $\operatorname{dim}_{\mathbb{R}} U=4 r n-8 r^{2} \equiv 0 \bmod 4$. For $d=1$, the quantity $r(2 n-2 r+1)$ may not be divisible by 4 . In this latter case, we take the larger parabolic associated to $\bullet \circ \bullet \bullet \bullet \cdots$, for which $\operatorname{dim}_{\mathbb{R}} U=\mu\left(A_{n}\right)-2 \mu\left(A_{1}\right)-A_{n-4}$. This calculation has been done in the rank 1 case (with $d=2$ ), and is congruent to $0 \bmod 4$.

Type $B_{n}$ or $C_{n}$ : Here $B_{n}$ is the special orthogonal group $\mathrm{SO}_{2 n+1}(k, q)$, where $q$ is a quadratic form of index $r$, and defect 1 in case char $k=2$. Here $C_{n}$ is the special unitary group $\mathrm{SU}_{2 n / d}(D, h)$, where $D$ is a division algebra of degree $d$ over $k$, and $h$ is a nondegenerate antihermitian sesquilinear form of index $r$ relative to an involution $\sigma$ of the first kind such that $D^{\sigma}$ (the space of symmetric elements) has dimension $\left(\begin{array}{l}d \\ 2\end{array}\right)$. When $d=1$, the group is simply $\operatorname{Sp}_{2 n}(k)$. For $C_{n}$, we have $d=2^{a} \mid 2 n$ and $d \geq 1$. If $d=1$, then $n=r$.

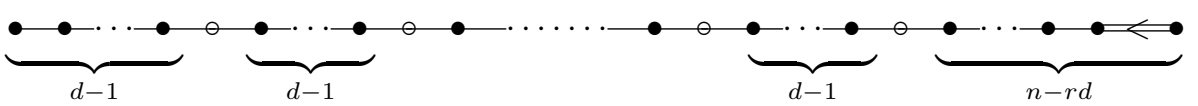

When $n=r d$, the right end becomes $\bullet \bullet 0$

(1) rank 1: $\circ \bullet \bullet \cdots$ for $d=1$ and $\bullet \circ \bullet \bullet \bullet \cdots$ for $d=2$

$$
\begin{aligned}
\operatorname{dim}_{\mathbb{R}} U & =\mu\left(B_{n}\right)-\mu\left(A_{d-1}\right)-\mu\left(B_{n-d}\right) \\
& =n^{2}-\left(\begin{array}{l}
d \\
2
\end{array}\right)-(n-d)^{2} \\
& =2 d n-d^{2}-\left(\begin{array}{l}
d \\
2
\end{array}\right) \\
& = \begin{cases}1 & \text { if } d \text { and } n \text { odd } \\
3 & \text { otherwise. }\end{cases}
\end{aligned}
$$

(2) $\operatorname{rank} 2$ :

(a) $\quad \circ \circ \bullet \bullet \cdots$ for $d=1$

(b)

$\operatorname{dim}_{\mathbb{R}} U=\mu\left(B_{n}\right)-\mu\left(B_{n-2}\right)=n^{2}-(n-2)^{2}=4 n-4 \equiv 0 \bmod 4$.

$\bullet \circ \bullet \circ \bullet \bullet \cdots$ if $d=2$

$\operatorname{dim}_{\mathbb{R}} U=\mu\left(B_{n}\right)-2 \mu\left(A_{1}\right)-\mu\left(B_{n-4}\right)=n^{2}-2(1)-(n-4)^{2}=8 n-18 \equiv 2$ $\bmod 4$.

Since we do not have $0 \bmod 4$, we compute larger parabolics by turning white nodes black. The case in which we fill in the leftmost white node 
has been computed in (1). The remaining case is

\section{$\bullet \bullet \bullet \bullet \bullet \bullet-\cdots$}

for which $\operatorname{dim}_{\mathbb{R}} U=\mu\left(B_{n}\right)-\mu\left(A_{3}\right)-\mu\left(B_{n-4}\right)=n^{2}-6-(n-4)^{2}=$ $8 n-22 \equiv 2 \bmod 4$.

(3) rank 3: We can assume $d=2$, since we have 0 above for $d=1$ (see 2a).

$\circ \circ \cdots \bullet \bullet \cdots$ for $d=1$ and $\bullet \circ \bullet \circ \bullet \circ \bullet \bullet \bullet \cdots$ for $d=2$

(a) For $d=1$, we immediately resort to the larger parabolic by blackening the third white dot. This case is computed in 2 a to yield $0 \bmod 4$.

(b) For $d=2$, we have $\operatorname{dim}_{\mathbb{R}} U=\mu\left(B_{n}\right)-3 \mu\left(A_{1}\right)-\mu\left(B_{n-6}\right)=n^{2}-$ $3(1)-(n-6)^{2}=12 n-39 \equiv 1 \bmod 4$. Since we do not have $0 \bmod$ 4 , we compute the dimension related to the higher parabolics that are previously unconsidered.

(i)

$$
\begin{aligned}
& \operatorname{dim}_{\mathbb{R}} U=\mu\left(B_{n}\right)-\mu\left(A_{5}\right)-\mu\left(B_{n-6}\right)=n^{2}-15-(n-6)^{2}= \\
& 12 n-51 \equiv 1 \bmod 4 . \\
& \text { (ii) } \quad \bullet \bullet \bullet \bullet \circ \bullet \bullet \bullet \cdot \text { or } \bullet \bullet \bullet \circ \bullet \bullet \bullet \bullet \cdot \cdots \\
& \operatorname{dim}_{\mathbb{R}} U=\mu\left(B_{n}\right)-\mu\left(A_{1}\right)-\mu\left(A_{3}\right)-\mu\left(B_{n-6}\right)=n^{2}-1-6-(n- \\
& 1)^{2}=12 n-43 \equiv 1 \bmod 4 .
\end{aligned}
$$

(4) rank 4: The $d=1$ can be handled as in (3a). We may then assume $d=2$. In this case, we immediately consider the larger parabolic obtained by blackening the three leftmost white nodes:

$$
\begin{aligned}
& \bullet \bullet \bullet \bullet \bullet \bullet \bullet \circ \bullet \bullet \bullet-\cdots \\
& \operatorname{dim}_{\mathbb{R}} U=\mu\left(B_{n}\right)-\mu\left(A_{7}\right)-\mu\left(B_{n-8}\right)=n^{2}-\left(\begin{array}{l}
8 \\
2
\end{array}\right)-(n-8)^{2}=16 n-92 \equiv 0 \\
& \bmod 4 .
\end{aligned}
$$

Type ${ }^{1} D_{n}$ : Over $\mathbb{Q}$ there are only the possibilities $d=1$ and 2 . If char $k \neq 2$, we have the special unitary group $\mathrm{SU}_{2 n / d}(D, h)$, where $D$ is a central division algebra of degree 2 over $k$, and $h$ is a nondegenerate Hermitian form of discriminant 1 and index $r$, relative to an involution $\sigma$ of the first kind such that $D^{\sigma}$ (the space of symmetric elements) has dimension $\left(\begin{array}{l}d \\ 2\end{array}\right)$. Here $d=2^{a} \mid 2 n$ and $d \geq 1$. Also $r d \leq n$ and $n \neq r d+1$.

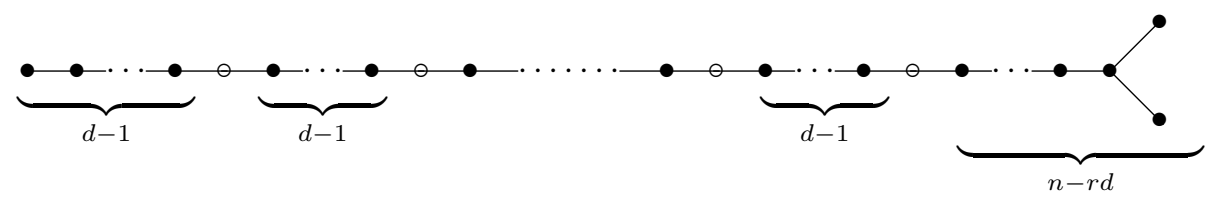

When $n-r d \leq 2$, the right end has one of the following forms. Note that the case $n=r d+1$ does not occur. In the cases (i) $(n, d)=(r, 1)$, (ii) $(n, d)=(2 r, 2)$, (iii) $n=r d$ and $d \geq 3$, (iv) $n=r d+2$, we have the following, respectively:
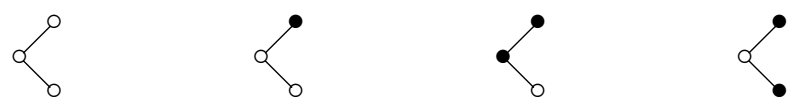
(1) rank 1:

$$
\begin{aligned}
& \bullet \bullet \cdots \text { for } d=1 \text { or } \bullet \cdots \cdots \text { for } d=2 \\
\operatorname{dim}_{\mathbb{R}} U & =\mu\left(D_{n}\right)-\mu\left(A_{d-1}\right)-\mu\left(D_{n-d}\right) \\
& =\left(n^{2}-n\right)-\left(\begin{array}{l}
d \\
2
\end{array}\right)-\left((n-d)^{2}-(n-d)\right) \\
& =2 n d-d^{2}-d-\left(\begin{array}{l}
d \\
2
\end{array}\right) \\
& = \begin{cases}1 & \text { if } d=2, \\
2 & \text { if } n \text { even and } d=1, \\
0 & \text { if } n \text { and } d \text { odd. }\end{cases}
\end{aligned}
$$

(2) rank 2: $\quad \circ \quad 0 \bullet \bullet \bullet \bullet \cdots$ for $d=1$ and $\bullet \circ \bullet \circ \bullet \bullet \bullet \cdots$ for $d=2$

$$
\begin{aligned}
\operatorname{dim}_{\mathbb{R}} U & =\mu\left(D_{n}\right)-2 \mu\left(A_{d-1}\right)-\mu\left(D_{n-2 d}\right) \\
& =\left(n^{2}-n\right)-2\left(\begin{array}{l}
d \\
2
\end{array}\right)-\left((n-2 d)^{2}-(n-2 d)\right) \\
& =4 n d-4 d^{2}-2 d-2\left(\begin{array}{l}
d \\
2
\end{array}\right) \\
& \equiv 2 d-2\left(\begin{array}{l}
d \\
2
\end{array}\right) \bmod 4 \\
& \equiv \begin{cases}2 \bmod 4 & \text { if } d=1, \\
2 \bmod 4 & \text { if } d=2 .\end{cases}
\end{aligned}
$$

Since we do not have $0 \bmod 4$, we consider the cases of larger parabolics.

$\bullet \circ \bullet \bullet \cdots$ for $d=1$ and $\bullet \bullet \bullet \bullet \bullet \cdots$ for $d=2$

$$
\begin{aligned}
\operatorname{dim}_{\mathbb{R}} U & =\mu\left(D_{n}\right)-\mu\left(A_{2 d-1}\right)-\mu\left(D_{n-2 d}\right) \\
& =\left(n^{2}-n\right)-\left(\begin{array}{c}
2 d \\
2
\end{array}\right)-\left((n-2 d)^{2}-(n-2 d)\right) \\
& \equiv \begin{cases}1 \bmod 4 & \text { if } d=1, \\
2 \bmod 4 & \text { if } d=2 .\end{cases}
\end{aligned}
$$

(3) rank 3: Given the situation in rank 1, we may assume that $d$ or $n$ is even. $\circ \quad 0 \quad 0 \bullet \bullet \bullet \cdots$ for $d=1$ and $\bullet \circ \bullet \circ \bullet \circ \bullet \bullet \bullet \cdots$ for $d=2$

$$
\begin{aligned}
\operatorname{dim}_{\mathbb{R}} U & =\mu\left(D_{n}\right)-3 \mu\left(A_{d-1}\right)-\mu\left(D_{n-3 d}\right) \\
& =\left(n^{2}-n\right)-3\left(\begin{array}{l}
d \\
2
\end{array}\right)-\left((n-6)^{2}-(n-6)\right) \\
& =6 d n-9 d^{2}-3 d-3\left(\begin{array}{l}
d \\
2
\end{array}\right) \\
& \equiv \begin{cases}0 \bmod 4 & \text { if } d=1 \text { (here } n \text { even), } \\
3 \bmod 4 & \text { if } d=2 .\end{cases}
\end{aligned}
$$

Given this calculation, we now compute the dimension for larger parabolics when $d=2$. We only list the cases that have not been previously computed. 
(a)

$$
\begin{aligned}
\bullet \bullet & \bullet \bullet \circ \bullet \bullet \bullet \cdots \text { for } d=2 \\
\operatorname{dim}_{\mathbb{R}} U & =\mu\left(D_{n}\right)-\mu\left(A_{5}\right)-\mu\left(D_{n-6}\right) \\
& =\left(n^{2}-n\right)-\left(\begin{array}{l}
6 \\
2
\end{array}\right)-\left((n-6)^{2}-(n-6)\right) \\
& =12 n-21 \equiv 3 \bmod 4 .
\end{aligned}
$$

(b)

for $d=2$

$$
\begin{aligned}
\operatorname{dim}_{\mathbb{R}} U & =\mu\left(D_{n}\right)-\mu\left(A_{1}\right)-\mu\left(A_{3}\right)-\mu\left(D_{n-6}\right) \\
& =\left(n^{2}-n\right)-\left(\begin{array}{l}
2 \\
2
\end{array}\right)-\left(\begin{array}{l}
4 \\
2
\end{array}\right)-\left((n-6)^{2}-(n-6)\right) \\
& =12 n-49 \equiv 3 \bmod 4 .
\end{aligned}
$$

(4) $\operatorname{rank} \geq 4$. We may assume that $d=2$.

$$
\begin{aligned}
\operatorname{dim}_{\mathbb{R}} U & =\mu\left(D_{n}\right)-\mu\left(D_{n-8}\right)-4 \mu\left(A_{1}\right) \\
& =\left(n^{2}-n\right)-\left((n-8)^{2}-(n-8)\right)-4\left(\begin{array}{l}
2 \\
2
\end{array}\right) \\
& =16 n-76 \equiv 0 \bmod 4 .
\end{aligned}
$$

Type ${ }^{2} D_{n}$ : The same as ${ }^{1} D_{n}$ except that all forms in question have now discriminant $\neq 1$. Here $d=2^{a} \mid 2 n$ and $d \geq 1$. Also $r d \leq n-1$.

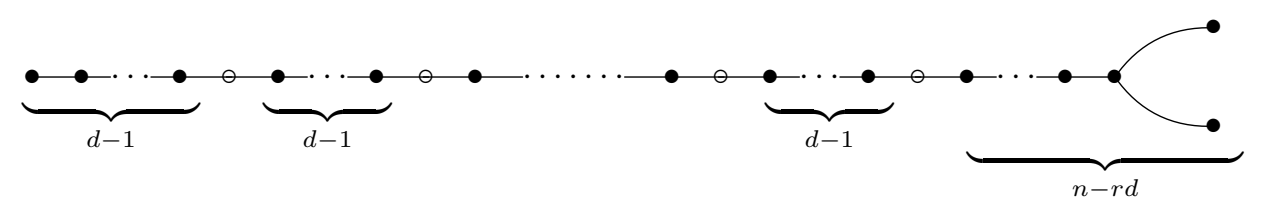

When $n=r d+1$, then $d=1$ or 2 , and the right end becomes respectively:
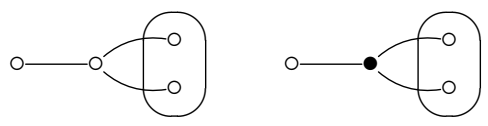

Calculations are the same as for ${ }^{1} D_{n}$ except that the Dynkin diagram is slightly different if $n=r d+1$, where $r$ is the rank under consideration. We have the following cases. Recall that the two rightmost white nodes are always clustered as one.

(1) When $d=1$, we have $r=n-1$. Now note that $\mu\left(A_{1}\right) \equiv 1 \bmod 4, \mu\left(A_{2}\right) \equiv 3$ $\bmod 4$ and $\mu\left(A_{3}\right) \equiv 2 \bmod 6$. Therefore $\operatorname{dim}_{\mathbb{R}} U=\mu\left(D_{n}\right)-\mu\left(A_{j}\right) \equiv 0$ $\bmod 4$ for an appropriate choice of $j \in\{0,1,2,3\}$.

(2) When $d=2$, we have $2 r=n-1$, so that $n$ is odd. 
(a) $r=2$ and $n=5$.

$$
\operatorname{dim}_{\mathbb{R}} U=\mu\left(D_{5}\right)-2 \mu\left(A_{1}\right)=20-2=18 \equiv 2 \bmod 4 .
$$

Since we do not have $0 \bmod 4$, we compute the dimension for the larger

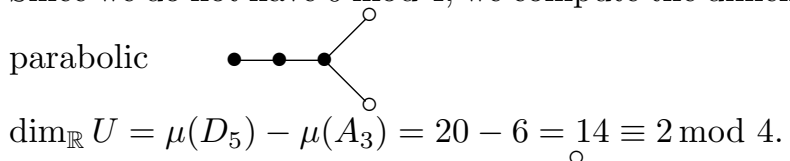

(b) $r=3$ and $n=7$.

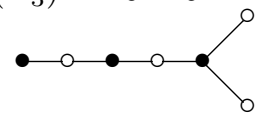

$$
\operatorname{dim}_{\mathbb{R}} U=\mu\left(D_{7}\right)-3 \mu\left(A_{1}\right)=42-3=39 \equiv 3 \bmod 4 .
$$

Since we do not have $0 \bmod 4$, we compute the dimension for larger parabolics.
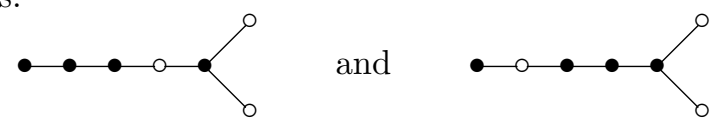

(ii)

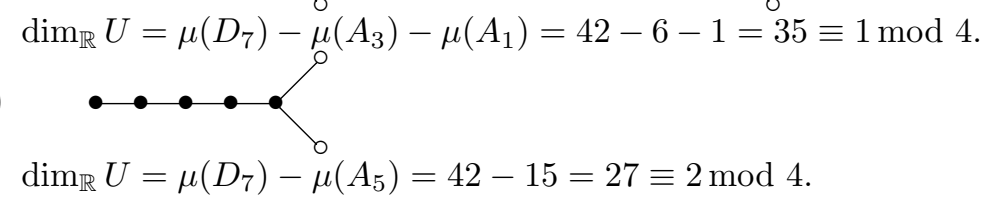

(c) Let $r \geq 4$. Then let $j$ be the least residue of $r \bmod 4$, and select the parabolic associated to $D_{2 r+1}-j A_{3}-(k-2 j) A_{1}$. Then we have $\operatorname{dim}_{\mathbb{R}} U=(2 r+1)^{2}-(2 r+1)-j\left(\begin{array}{l}3 \\ 2\end{array}\right)-(r-2 j)=4 k^{2}+k-j \equiv 0 \bmod 4$.

Type ${ }^{3,6} D_{4}$ : The subtype involving $D_{n}$ when $n=4$ requires special consideration.

(1) $\operatorname{rank} 1: \quad \circ:$

$$
\operatorname{dim}_{\mathbb{R}} U=\mu\left(D_{4}\right)-3 \mu\left(A_{1}\right)=12-3=1 \equiv 1 \bmod 4 .
$$

(2) rank 2: $\left.\begin{array}{r}0 \\ 0 \\ 0 \\ 0\end{array}\right) \quad$ Note that here we have the Borel subgroup.

$$
\operatorname{dim}_{\mathbb{R}} U=\mu\left(D_{4}\right)=12 \equiv 0 \bmod 4 .
$$

Type ${ }^{1} E_{6}$ : Here ${ }^{1} E_{6.2}^{28}$ is the form which can be realized as a collineation group of a Cayley plane, and ${ }^{1} E_{6,2}^{16}$ is the form constructed by means of an associative division algebra of degree 3 . There is nothing of rank 1 .

(1) first group of rank 2

$$
\operatorname{dim}_{\mathbb{R}} U=\mu\left(E_{6}\right)-\mu\left(D_{4}\right)=36-12=24 \equiv 0 \bmod 4 .
$$

(2) second group of rank $2 \quad \bullet \quad \longrightarrow$

$$
\operatorname{dim}_{\mathbb{R}} U=\mu\left(E_{6}\right)-2 \mu\left(A_{2}\right)=36-2(3)=30 \equiv 2 \bmod 4 .
$$

Since we do not have $0 \bmod 4$, we consider larger parabolics.

(a)

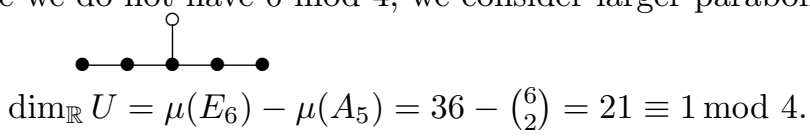


(b)

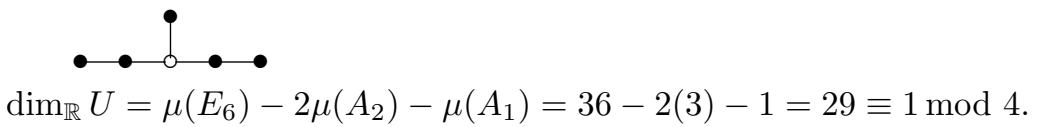

(3) In higher rank cases, we can always pass to the larger parabolic associated to the first group of rank 2 (Case 1).

Type ${ }^{2} E_{6}$ : There are five subtypes in this case.

(1) first group of rank 1

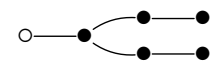

$$
\operatorname{dim}_{\mathbb{R}} U=\mu\left(E_{6}\right)-\mu\left(A_{5}\right)=36-\left(\begin{array}{l}
6 \\
2
\end{array}\right)=21 \equiv 1 \bmod 4 .
$$

(2) second group of rank 1

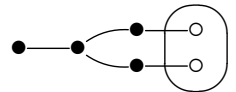

$$
\operatorname{dim}_{\mathbb{R}} U=\mu\left(E_{6}\right)-\mu\left(D_{4}\right)=36-12=24 \equiv 0 \bmod 4 .
$$

(3) first group of rank 2

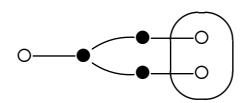

Here we resort to the larger parabolic associated with the diagram in Case 2 above and obtain $0 \bmod 4$.

(4) second group of rank 2

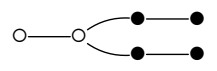

This calculation has been done in the case of ${ }^{1} E_{6}$ in the second group of rank 2 , in which we obtain 1 or $2 \bmod 4$.

(5) higher rank:

$$
\operatorname{dim}_{\mathbb{R}} U=\mu\left(E_{6}\right)=36 \equiv 0 \bmod 4 .
$$

Type $E_{7}$ : Here $E_{7,3}^{28}$ is the form constructed by means of a Cayley division algebra. There are no subtypes of rank 1.

(1) $\operatorname{rank} 2: \quad \bullet \circ \bullet \bullet \bullet \circ$

$$
\operatorname{dim}_{\mathbb{R}} U=\mu\left(E_{7}\right)-\mu\left(A_{1}\right)-\mu\left(D_{4}\right)=63-1-\left(4^{2}-4\right)=50 \equiv 2 \bmod 4 .
$$

Since we do not have $0 \bmod 4$, we consider larger parabolics.

(a)

$$
\begin{aligned}
& \bullet \bullet \bullet \bullet \\
& \operatorname{dim}_{\mathbb{R}} U=\mu\left(E_{7}\right)-\mu\left(D_{6}\right)=63-\left(6^{2}-6\right)=33 \equiv 1 \bmod 4 . \\
& \text { (b) } \bullet \circ \bullet \bullet \bullet \bullet \\
& \operatorname{dim}_{\mathbb{R}} U=\mu\left(E_{7}\right)-\mu\left(A_{1}\right)-\mu\left(D_{5}\right)=63-1-\left(5^{2}-5\right)=42 \equiv 2 \bmod 4 .
\end{aligned}
$$

(2) $\operatorname{rank} 3: \quad \circ \quad \cdots \bullet \bullet \bullet$

$$
\operatorname{dim}_{\mathbb{R}} U=\mu\left(E_{7}\right)-\mu\left(D_{4}\right)=63-\left(4^{2}-4\right)=51 \equiv 3 \bmod 4 .
$$

Since we do not have $0 \bmod 4$, we consider larger parabolics.

(a)

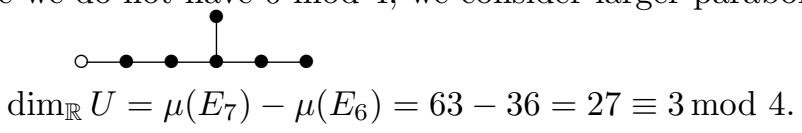


(b)

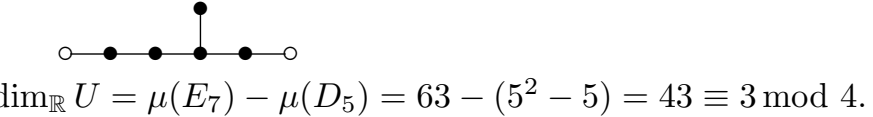

(c) The remaining case is handled in (1).

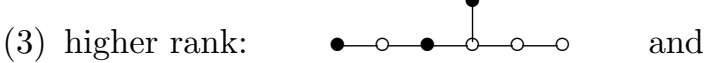
$\operatorname{dim}_{\mathbb{R}} U=\mu\left(E_{7}\right)-3 \mu\left(A_{1}\right)=63-3=60 \equiv 0 \bmod 4$.

Type $E_{8}$ : Here $E_{8,4}^{28}$ is the form constructed by means of a Cayley division algebra. There are only two subtypes under consideration:

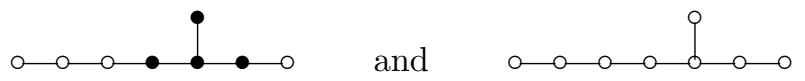

$\operatorname{dim}_{\mathbb{R}} U=\mu\left(E_{8}\right)-\mu\left(D_{4}\right)=120-\left(4^{2}-4\right)=108 \equiv 0 \bmod 4$.

Type $F_{4}$ : A group of this type is always the automorphism group of an exceptional simple Jordan algebra $J$. There are only two subtypes under consideration.

(1) rank 1: • $\Longrightarrow$ $\operatorname{dim}_{\mathbb{R}} U=\mu\left(F_{4}\right)-\mu\left(B_{3}\right)=24-3^{2}=15 \equiv 3 \bmod 4$.

(2) higher rank: $\Longrightarrow$ $\operatorname{dim}_{\mathbb{R}} U=\mu\left(F_{4}\right)=24 \equiv 0 \bmod 4$.

Type $G_{2}$ : A group of type $G_{2}$ is always the automorphism group of a Cayley algebra.

rank 2: $\Longrightarrow 0 \quad \operatorname{dim}_{\mathbb{R}} U=\mu\left(G_{2}\right)=6 \equiv 2 \bmod 4$.

Since we do not have $0 \bmod 4$, we compute the case of the larger parabolic $\Longleftrightarrow$ for which $\operatorname{dim}_{\mathbb{R}} U=\mu\left(G_{2}\right)-\mu\left(A_{1}\right)=6-1=5 \equiv 1 \bmod 4$.

Conclusion: When the $\mathbb{Q}$-rank is at least 4 , there is always a parabolic subgroup for which the real dimension of the unipotent radical is divisible by 4 . When $\mathbb{Q}$-rank is 3 , there are some exceptions.

Remark 6.1. There is a rank-3 form of $E_{7}$ that does not have a unipotent radical whose dimension is divisible by 4 . This $\mathbb{Q}$-form of $G$ could be split over $\mathbb{R}$. So both the Hermitian symmetric real form and the split real form of $E_{7}$ have arithmetic lattices in which no nontrivial horospherical $\mathbb{Q}$-subgroup has dimension divisible by 4. In the Hermitian case, the structure set is nontrivial and infinite, but we do not know more.

\section{REFERENCES}

1. D. W. Anderson and Luke Hodgkin, The K-theory of Eilenberg-MacLane complexes, Topology 7 (1968), 317-329.

2. Avner Ash, Non-square-integrable cohomology of arithmetic groups, Duke Math. J. 47 (1980), no. 2, 435-449.

3. Avner Ash and Armand Borel, Generalized modular symbols, Cohomology of arithmetic groups and automorphic forms (Luminy-Marseille, 1989), Lecture Notes in Math., vol. 1447, Springer, Berlin, 1990, pp. 57-75. 
4. Avner Ash, Paul E. Gunnells, and Mark McConnell, Cohomology of congruence subgroups of $\mathrm{SL}_{4}(\mathbb{Z})$, J. Number Theory 94 (2002), no. 1, 181-212.

5. W. L. Baily, Jr. and A. Borel, Compactification of arithmetic quotients of bounded symmetric domains, Ann. of Math. (2) 84 (1966), 442-528.

6. Arthur Bartels and Wolfgang Lück, Induction theorems and isomorphism conjectures for $K$ and L-theory, Forum Math. 19 (2007), no. 3, 379-406.

7. Arthur Bartels, Wolfgang Lück, Holger Reich, and Henrik Rüping, $k$ - and $l$ - theory of group rings over $\mathrm{GL}_{n}(\mathbb{Z})$.

8. Bachir Bekka, Pierre de la Harpe, and Alain Valette, Kazhdan's property (T), New Mathematical Monographs, vol. 11, Cambridge University Press, Cambridge, 2008.

9. Jonathan Block and Shmuel Weinberger, Arithmetic manifolds of positive scalar curvature, J. Differential Geom. 52 (1999), no. 2, 375-406.

10. A. Borel and J.-P. Serre, Corners and arithmetic groups, Comment. Math. Helv. 48 (1973), 436-491, Avec un appendice: Arrondissement des variétés à coins, par A. Douady et L. Hérault.

11. Armand Borel, Stable real cohomology of arithmetic groups, Ann. Sci. École Norm. Sup. (4) 7 (1974), 235-272 (1975).

12. Cohomology of arithmetic groups, Proceedings of the International Congress of Mathematicians (Vancouver, B.C., 1974), Vol. 1, Canad. Math. Congress, Montreal, Que., 1975, pp. $435-442$.

13. Armand Borel and Harish-Chandra, Arithmetic subgroups of algebraic groups, Ann. of Math. (2) 75 (1962), 485-535.

14. Armand Borel and Lizhen Ji, Compactifications of symmetric and locally symmetric spaces, Mathematics: Theory \& Applications, Birkhäuser Boston Inc., Boston, MA, 2006.

15. Henri Cartan, Séminaire Henri Cartan, 12ième année: 1959/60. Périodicité des groupes d'homotopie stables des groupes classiques, d'après Bott, Deux fascicules. Deuxième édition, corrigée, École Normale Supérieure. Secrétariat mathématique, Paris, 1961. Fasc. 1 (Exp. 1-9), 1961.

16. Stanley S. Chang, Coarse obstructions to positive scalar curvature in noncompact arithmetic manifolds, J. Differential Geom. 57 (2001), no. 1, 1-21.

17. Stanley S. Chang and Shmuel Weinberger, On Novikov-type conjectures, Surveys in noncommutative geometry, Clay Math. Proc., vol. 6, Amer. Math. Soc., Providence, RI, 2006, pp. $43-70$.

18. _ Topological nonrigidity of nonuniform lattices, Comm. Pure Appl. Math. 60 (2007), no. 2, 282-290.

19. Ruth Charney, On the problem of homology stability for congruence subgroups, Comm. Algebra 12 (1984), no. 17-18, 2081-2123.

20. Jeff Cheeger and Mikhael Gromov, $L_{2}$-cohomology and group cohomology, Topology 25 (1986), no. 2, 189-215.

21. Thomas Church and Benson Farb, In preparation.

22. Benson Farb and Peter B. Shalen, Real-analytic, volume-preserving actions of lattices on 4-manifolds, C. R. Math. Acad. Sci. Paris 334 (2002), no. 11, 1011-1014.

23. F. T. Farrell and W. C. Hsiang, The stable topological-hyperbolic space form problem for complete manifolds of finite volume, Invent. Math. 69 (1982), no. 1, 155-170.

24. F. T. Farrell and L. E. Jones, Rigidity for aspherical manifolds with $\pi_{1} \subset \mathrm{GL}_{m}(\mathbf{R})$, Asian J. Math. 2 (1998), no. 2, 215-262.

25. Jens Franke, A topological model for some summand of the Eisenstein cohomology of congruence subgroups, Eisenstein series and applications, Progr. Math., vol. 258, Birkhäuser Boston, Boston, MA, 2008, pp. 27-85.

26. Damien Gaboriau, Invariants $l^{2}$ de relations d'équivalence et de groupes, Publ. Math. Inst. Hautes Études Sci. (2002), no. 95, 93-150.

27. M. Gromov, Almost flat manifolds, J. Differential Geom. 13 (1978), no. 2, 231-241. 
28. Mikhael Gromov, Groups of polynomial growth and expanding maps, Inst. Hautes Études Sci. Publ. Math. (1981), no. 53, 53-73.

29. Sigurdur Helgason, Differential geometry and symmetric spaces, Pure and Applied Mathematics, Vol. XII, Academic Press, New York, 1962.

30. James E. Humphreys, Introduction to Lie algebras and representation theory, Graduate Texts in Mathematics, vol. 9, Springer-Verlag, New York, 1978, Second printing, revised.

31. L. Ji and R. MacPherson, Geometry of compactifications of locally symmetric spaces, Ann. Inst. Fourier (Grenoble) $\mathbf{5 2}$ (2002), no. 2, 457-559.

32. J. Jost and S.-T. Yau, On the rigidity of certain discrete groups and algebraic varieties, Math. Ann. 278 (1987), no. 1-4, 481-496.

33. Robion C. Kirby and Laurence C. Siebenmann, On the triangulation of manifolds and the Hauptvermutung, Bull. Amer. Math. Soc. 75 (1969), 742-749.

34. Ronnie Lee and R. H. Szczarba, On the homology and cohomology of congruence subgroups, Invent. Math. 33 (1976), no. 1, 15-53.

35. Alexander Lubotzky, On finite index subgroups of linear groups, Bull. London Math. Soc. 19 (1987), no. 4, 325-328.

36. Ib Madsen and R. James Milgram, The classifying spaces for surgery and cobordism of manifolds, Annals of Mathematics Studies, vol. 92, Princeton University Press, Princeton, N.J., 1979.

37. G. A. Margulis, Discrete subgroups of semisimple Lie groups, Ergebnisse der Mathematik und ihrer Grenzgebiete (3) [Results in Mathematics and Related Areas (3)], vol. 17, SpringerVerlag, Berlin, 1991.

38. Yozô Matsushima, On Betti numbers of compact, locally sysmmetric Riemannian manifolds, Osaka Math. J. 14 (1962), 1-20.

39. Martin Olbrich, $L^{2}$-invariants of locally symmetric spaces, Doc. Math. 7 (2002), 219-237 (electronic).

40. A. L. Onishchik and È. B. Vinberg, Lie groups and algebraic groups, Springer Series in Soviet Mathematics, Springer-Verlag, Berlin, 1990, Translated from the Russian and with a preface by D. A. Leites.

41. Vladimir Platonov and Andrei Rapinchuk, Algebraic groups and number theory, Pure and Applied Mathematics, vol. 139, Academic Press Inc., Boston, MA, 1994, Translated from the 1991 Russian original by Rachel Rowen.

42. Gopal Prasad, Strong rigidity of Q-rank 1 lattices, Invent. Math. 21 (1973), 255-286.

43. Gopal Prasad and M. S. Raghunathan, Cartan subgroups and lattices in semi-simple groups, Ann. of Math. (2) 96 (1972), 296-317.

44. I. Satake, Classification theory of semi-simple algebraic groups, Marcel Dekker Inc., New York, 1971, With an appendix by M. Sugiura, Notes prepared by Doris Schattschneider, Lecture Notes in Pure and Applied Mathematics, 3.

45. Joachim Schwermer, Holomorphy of Eisenstein series at special points and cohomology of arithmetic subgroups of $\mathrm{SL}_{n}(\mathbf{Q})$, J. Reine Angew. Math. 364 (1986), 193-220.

46. Laurence C. Siebenmann, The obstruction to finding a boundary for an open manifold of dimension greater than 5, ProQuest LLC, Ann Arbor, MI, 1965, Thesis (Ph.D.)-Princeton University.

47. J. Tits, Classification of algebraic semisimple groups, Algebraic Groups and Discontinuous Subgroups (Proc. Sympos. Pure Math., Boulder, Colo., 1965), Amer. Math. Soc., Providence, R.I., 1966, 1966, pp. 33-62.

48. C. T. C. Wall, Surgery on compact manifolds, Academic Press, London, 1970, London Mathematical Society Monographs, No. 1.

49. B. A. F. Wehrfritz, Infinite linear groups. An account of the group-theoretic properties of infinite groups of matrices, Springer-Verlag, New York, 1973, Ergebnisse der Matematik und ihrer Grenzgebiete, Band 76.

50. Shmuel Weinberger, The Lipschitz rationality of linear representations, Comm. Pure Appl. Math. 40 (1987), no. 5, 609-610. 
51. Boris Weisfeiler, Strong approximation for Zariski-dense subgroups of semisimple algebraic groups, Ann. of Math. (2) 120 (1984), no. 2, 271-315.

52. David Witte Morris, Introduction to arithmetic groups.

53. Robert J. Zimmer, Ergodic theory and semisimple groups, Monographs in Mathematics, vol. 81, Birkhäuser Verlag, Basel, 1984.

Department of Mathematics, Wellesley College, Wellesley, MA 02481

E-mail address: schang@wellesley.edu

Department of Mathematics, University of Chicago, Chicago, IL 60637

E-mail address: shmuel@math.uchicago.edu 